BULLETIN (New Series) OF THE AMERICAN MATHEMATICAL SOCIETY

Volume 35, Number 4, October 1998, Pages 271-317

S 0273-0979(98)00765-4

\title{
ELLIPTIC ALGEBRO-GEOMETRIC SOLUTIONS OF THE KDV AND AKNS HIERARCHIES - AN ANALYTIC APPROACH
}

\author{
FRITZ GESZTESY AND RUDI WEIKARD
}

\begin{abstract}
We provide an overview of elliptic algebro-geometric solutions of the KdV and AKNS hierarchies, with special emphasis on Floquet theoretic and spectral theoretic methods. Our treatment includes an effective characterization of all stationary elliptic KdV and AKNS solutions based on a theory developed by Hermite and Picard.
\end{abstract}

\section{INTRODUCTION}

The story of J. Scott Russell chasing a soliton for a mile or two along the Edinburgh-Glasgow channel in 1834 has been told many times. It is the starting point of more than 160 years of an exciting history embracing a variety of deep mathematical ideas ranging from applied mathematics to algebraic geometry, Lie groups, and differential geometry. In this article we want to tell a certain aspect of this story, predominantly from an analytic point of view with special emphasis on Floquet theoretic and spectral theoretic methods. Other aspects of this story have recently been told by Lax [146], Palais [172], and Terng and Uhlenbeck [209].

We are interested in the class of evolution equations which permit being cast into the form $L_{t}=[P, L]$, where $(P, L)$ is a pair of operators, a so-called Lax pair, and $[P, L]$ denotes their commutator. Typically, for a fixed operator $L$, there is a sequence of operators $P$ such that $L_{t}=[P, L]$ defines an evolution equation. Hence we are actually concerned with hierarchies of evolution equations. Stationary solutions of such equations, corresponding to commuting operators $P$ and $L$, are related to algebraic curves and are therefore called algebro-geometric solutions. The stationary solutions of higher-order equations in the hierarchy play a decisive role in the study of the Cauchy problem for the lower-order equations in the hierarchy. Therefore, and because of the connection to algebraic geometry, the stationary problem has drawn considerable attention.

The central object of this article is the class of elliptic algebro-geometric solutions of the KdV and AKNS hierarchies (cf. Section 2.3). In a sense, this class represents a natural generalization of the class of soliton solutions and enjoys a much richer structure due to its connections to algebraic geometry inherent in its construction. However, while frequently the algebraic aspects of this construction dominate the stage, we will purposely portray a different analytical view often neglected (and to

Received by the editors May 20, 1998, and in revised form August 10, 1998.

1991 Mathematics Subject Classification. Primary 34L40, 35Q53, 35Q55; Secondary 34B30, 34L05, 35Q51.

Research supported in part by the US National Science Foundation under Grant Nos. DMS9401816 and DMS-9623121.

(C) 1998 by the authors 
some extent forgotten) in this context. Consequently, we will focus in the following on the interplay between spectral (and Floquet) theoretic properties of the Lax pairs (cf. Section 2.3) defining the integrable evolution equations in question on the one hand, and the construction and properties of the underlying compact Riemann surface associated with algebro-geometric solutions on the other.

In our quest to characterize the class of elliptic algebro-geometric solutions of soliton equations in an effective manner, we rely heavily on a marvelous theory developed by Fuchs, Halphen, Hermite, Mittag-Leffler, and especially, Picard. We have chosen to provide a rather extensive bibliography concerning this classical work since some of the cornerstones of this theory seem to have been forgotten, and at times appear to be independently rediscovered (cf. Subsection 2.9 and the bibliographical remarks at the end of Remark 3.9). In particular, Picard's theorem (Theorem 2.7), a key in the aforementioned characterization problem, apparently had not been used in the extensive body of literature surrounding elliptic algebrogeometric solutions of various hierarchies of soliton equations. Picard's theorem suggests one consider the independent variable of the differential equation in question as a complex variable and study the consequences of assuming the existence of a meromorphic fundamental system of solutions. This led to the discovery of a connection between the existence of such a meromorphic fundamental system of solutions of a linear differential equation $L y=z y$ for all values of $z$ and algebrogeometric properties of $L$. In particular, it connects the existence of a meromorphic fundamental system of solutions of $L y=z y$ to the integrability of the nonlinear equations associated with $L$ via the Lax pair formalism, as we demonstrated in the KdV and AKNS cases (cf. Theorems 3.12 and 4.8). As it turns out, Picard was describing solutions of differential equations with elliptic coefficients, which represent simultaneous Floquet solutions with respect to all fundamental periods of the underlying period lattice of the torus in question. Hence Floquet theory, and consequently, spectral theory, naturally enters when analyzing Lax pairs for completely integrable evolution equations and their elliptic algebro-geometric solutions.

Section 2 provides an introduction into the KdV hierarchy and its elliptic algebrogeometric solutions, reviews the necessary Floquet and spectral theoretic background for Hill (Lax) operators, presents Picard's theorem on linear differential equations with elliptic coefficients and, especially, provides a historical perspective of the subject. Section 3 is devoted to the KdV hierarchy and its stationary solutions. The case of rational, periodic, and elliptic stationary KdV solutions is described in detail and the role of meromorphic fundamental systems of solutions for the corresponding Lax operator associated with stationary KdV solutions is underscored. This section culminates in an explicit characterization of all elliptic, simply periodic, and rational stationary KdV solutions (see [96], [97], [233] for the original results). Our final Section 4 describes analogous results for the AKNS hierarchy; in particular, it contains an effective characterization of all elliptic algebro-geometric AKNS solutions (cf. [98] for the original proof). We hope the enormous bibliography at the end (still necessarily incomplete) will lose some of its intimidating character once the reader begins to appreciate the large body of knowledge amassed by some of the giants in the field, starting with Hermite.

Finally we remark that connections between completely integrable systems and Seiberg-Witten theory via elliptic Calogero-Moser-type models have recently led to a strong resurgence of the field of elliptic solutions of soliton equations. It seems difficult to keep up with all the current activities in the corresponding preprint 
archives. Hence we refer, for instance, to Babelon and Talon [12], Donagi and Markman [46], Donagi and Witten [47], Itoyama and Morozov [119], Krichever [139], Krichever, Babelon, Billey, and Talon [140], Krichever and Phong [141], Krichever, Wiegmann, and Zabrodin [142], Krichever and Zabrodin [143], Kuznetsov, Nijhoff, and Sklyanin [144], Levin and Olshanetsky [148], Marshakov [151], and Vaninsky [228]), from which the interested reader can easily find further sources.

\section{BACKGROUND AND SOME HISTORY}

2.1. The Korteweg-de Vries Equation. In the first few decades after their discovery, solitary waves were considered, for instance, by Stokes, Boussinesq, and Lord Rayleigh. But the most far reaching and, in a sense, lasting contribution was made in 1895 by Korteweg and de Vries [128], who deduced their celebrated equation, which may be cast in the form

$$
q_{t}=\frac{1}{4} q_{x x x}+\frac{3}{2} q q_{x} .
$$

(However, as recently alluded to by Pego [177], Boussinesq originally noted a system of equations equivalent to (2.1.1) and used it to study solitary waves in the 1870's.) In 1960 Gardner and Morikawa [78] used the KdV equation to describe collisionless-plasma magnetohydromagnetic waves. Since then the KdV equation has been rederived again and again in different contexts as a model equation describing a considerable variety of physical phenomena, and can now be considered one of the basic equations in mathematical physics. Even more important is the fact that this development triggered the examination of a whole class of other nonlinear evolution equations, some of which are of great physical relevance, such as the nonlinear Schrödinger equation, the sine-Gordon equation, the Toda lattice, the Boussinesq equation, the Kadomtsev-Petviashvili equation, etc.

But the description of wave phenomena alone would not have turned the subject into the kind of industry it is today. In 1955 Fermi, Pasta, and Ulam [66] studied a system of nonlinear oscillators which may be viewed as a discretized version of the KdV equation on a finite interval with periodic boundary conditions. To their surprise they found that energy shows little tendency toward equipartition among the degrees of freedom. Later Kruskal and Zabusky [240] examined the $\mathrm{KdV}$ equation numerically and observed the formation of solitary waves which, using their words, "pass through one another without losing their identity." In order to emphasize this particle-like behavior Kruskal and Zabusky coined the term soliton.

In 1968 Miura [163] introduced the transformation

$$
q(x, t)=z-v(z, x, t)^{2}-v_{x}(z, x, t)
$$

in which $z$ is a (generally complex-valued) spectral parameter. This transformation, now known as Miura's transformation, relates the KdV equation to a variant of the so-called modified $\mathrm{KdV}(\mathrm{mKdV})$ equation,

$$
v_{t}=v_{x x x}-6 v^{2} v_{x}+6 z v_{x} .
$$

Since (2.1.2) is a Riccati equation for $v$, it may be transformed into the linear equation

$$
(L(t) y)(z, x, t)=y^{\prime \prime}(z, x, t)+q(x, t) y(z, x, t)=z y(z, x, t)
$$


by introducing $v(z, x, t)=y^{\prime}(z, x, t) / y(z, x, t)$ (primes denote derivatives with respect to $x$ ). Gardner, Kruskal, and Miura [164] showed that the eigenvalues of the $L^{2}(\mathbb{R})$-operator associated with $L(t)$ do not depend on $t$; that is, they are constants of the motion under the KdV flow. This was the starting point for another seminal work, this time by Gardner, Greene, Kruskal, and Miura [76], in which they used the inverse scattering method to solve the Cauchy problem for the KdV equation with rapidly decaying initial data. This method, which represents a nonlinear ana$\log$ of the Fourier transform to solve linear partial differential equations, initially consists of computing the scattering data of $L(0)$, then propagating them in time (which is simple), and finally reconstructing the potential $q(x, t)$ in $L(t)$ via the Gelfand-Levitan, or rather, the Marchenko equation. Miraculously, this function $q(x, t)$ is the desired solution of the Cauchy problem. See, for instance, Ablowitz and Clarkson [1], Ch. 2; Asano and Kato [11], Chs. 5,6; Dodd, Eilbeck, Gibbon, and Morris [45], Ch. 4; Drazin and Johnson [51], Ch. 4; Gardner, Greene, Kruskal, and Miura [77]; Iliev, Khristov, and Kirchev [116], Ch. 3; Lax [146]; Marchenko [152], Ch. 4; and Palais [172] for more details.

2.2. Hamiltonian Systems. The dynamics of a classical mechanical particle system is described as a flow on a symplectic space, called the phase space. The phase space is the cotangent bundle of a Riemannian manifold, called the configuration space, describing the positions of the particles. The flow is given by Hamilton's equation $q_{t}=\operatorname{grad}_{S}(H)$, where $\operatorname{grad}_{S}$ is a symplectic gradient and $H$ is the socalled Hamiltonian of the system. If there are only finitely many, say $n$, degrees of freedom (as in a system of finitely many interacting particles), then $q=(\xi, \eta)$, where $\xi$ is a vector of local coordinates on the configuration space (called generalized coordinates) and $\eta$ is the vector of the associated momenta (called generalized momenta). $H$ is then a function of $\xi, \eta$, and $t$, and the symplectic gradient is given by

$$
\operatorname{grad}_{S}=\left(\begin{array}{cc}
0 & I_{n} \\
-I_{n} & 0
\end{array}\right) \operatorname{grad}_{(\xi, \eta)},
$$

where $I_{n}$ is the $n \times n$ identity matrix. A canonical transformation is a change of coordinates in phase space which leaves the form of Hamilton's equation invariant. Sometimes there exists a canonical transformation such that the transformed Hamiltonian is a function of the transformed generalized momenta alone which are then called action variables while the transformed generalized coordinates are called angle variables. The action variables are then constants of the motion while the angle variables change linearly with time. If this happens, the system is called completely integrable.

In 1968 Gardner, Kruskal, and Miura [164] explicitly constructed an infinite sequence of constants of the motion providing the first hint toward a Hamiltonian structure of the KdV equation. Next, in 1971, Faddeev and Zakharov [241], in an attempt to explain the unusual behavior of the KdV equation, showed that it can be viewed as an infinite-dimensional completely integrable Hamiltonian system. Take, for instance, the Schwartz space $\mathcal{S}(\mathbb{R})$ as phase space which can be viewed as a symplectic manifold. A symplectic gradient on $\mathcal{S}(\mathbb{R})$ is given by

$$
\operatorname{grad}_{S}=\frac{\partial}{\partial x} \operatorname{grad},
$$


where $\operatorname{grad}(F)$ denotes the Lagrangian (or variational) derivative of $F: \mathcal{S}(\mathbb{R}) \rightarrow \mathbb{R}$. More precisely, if $F$ is of the form

$$
F(q)=\int_{-\infty}^{\infty} d x \widetilde{F}\left(q, q^{\prime}, \ldots, q^{(n)}\right),
$$

where $\widetilde{F}: \mathbb{R}^{n+1} \rightarrow \mathbb{R}$ is a polynomial function without a constant term, then

$$
\operatorname{grad}(F)=\frac{\partial \widetilde{F}}{\partial q}-\left(\frac{\partial \widetilde{F}}{\partial q^{\prime}}\right)^{\prime}+\cdots+(-1)^{n}\left(\frac{\partial \widetilde{F}}{\partial q^{(n)}}\right)^{(n)}
$$

and hence

$$
\operatorname{grad}_{S}(F)=\left(\frac{\partial \widetilde{F}}{\partial q}\right)^{\prime}-\left(\frac{\partial \widetilde{F}}{\partial q^{\prime}}\right)^{\prime \prime}+\cdots+(-1)^{n}\left(\frac{\partial \widetilde{F}}{\partial q^{(n)}}\right)^{(n+1)}
$$

using primes to denote derivatives with respect to $x$. The Hamiltonian flow

$$
\dot{q}=\operatorname{grad}_{S}(F)
$$

then becomes the nonlinear evolution equation

$$
q_{t}=\left(\frac{\partial \widetilde{F}}{\partial q}\right)^{\prime}-\left(\frac{\partial \widetilde{F}}{\partial q^{\prime}}\right)^{\prime \prime}+\cdots+(-1)^{n}\left(\frac{\partial \widetilde{F}}{\partial q^{(n)}}\right)^{(n+1)} .
$$

In the special case of the $\mathrm{KdV}$ flow, the Hamiltonian $H$ is given by

$$
H: \mathcal{S}(\mathbb{R}) \rightarrow \mathbb{R}, \quad q \mapsto \frac{1}{4} \int_{-\infty}^{\infty} d x\left(q(x)^{3}-\frac{1}{2} q^{\prime}(x)^{2}\right),
$$

and Hamilton's equation $q_{t}=\operatorname{grad}_{S}(H)$ results in the KdV equation (2.1.1). Analogous considerations apply to the periodic case replacing $\int_{-\infty}^{\infty} d x$ by $\int_{0}^{\Omega} d x$, with $\Omega>0$ the fundamental period of $q$. We refer, for instance, to Palais [172] for more information.

2.3. Lax Pairs and the KdV Hierarchy. Gardner, Greene, Kruskal, and Miura [76] had shown that the $L^{2}$-spectrum of $L(t)=\partial^{2} / \partial x^{2}+q(x, t)$ is independent of $t$ whenever $q$ is a solution of the $\mathrm{KdV}$ equation. This discovery inspired Lax to conjecture that the operators $L(t)$ are all unitarily equivalent to one another; that is, $L(0)=U(t)^{-1} L(t) U(t)$ for some family of unitary operators $U(t)$. This then led to his celebrated commutator representation [145],

$$
q_{t}=L_{t}=\left[P_{3}, L\right],
$$

where $P_{3}$ denotes the third-order ordinary differential expression defined by

$$
P_{3}=\frac{1}{4} \frac{\partial^{3}}{\partial x^{3}}+\frac{3}{2} q \frac{\partial}{\partial x}+\frac{3}{4} q_{x}
$$

which governs the time evolution of $U(t)$ according to $U_{t}(t)=P_{3}(t) U(t), U(0)=I$. Indeed, the latter equation implies $\left(U(t)^{-1}\right)_{t}=-U(t)^{-1} P_{3}(t)$ and hence formally,

$$
\frac{d}{d t}\left(U(t)^{-1} L(t) U(t)\right)=U(t)^{-1}\left(L_{t}(t)-\left[P_{3}(t), L(t)\right]\right) U(t)=0
$$

yields

$$
U(t)^{-1} L(t) U(t)=L(0), \quad t \in \mathbb{R} .
$$

(Functional analytic arguments guaranteeing self-adjointness of $L(t)$ and unitarity of $U(t)$ in the Hilbert space $L^{2}(\mathbb{R} ; d x)$ can easily be supplied.) The letters $P$ and 
$L$ were first used by Gelfand and Dickey [83] in honor of Peter Lax. Accordingly, $\left(P_{3}, L\right)$ is called a Lax pair.

In fact, Lax designed his procedure for a far more general setting: it applies to evolution equations for $q$ whenever there is a one-to-one correspondence between $q(\cdot, t)$ and a (formally) symmetric operator $L(t)$. The goal is then to find a (formally) skew-symmetric operator $P(t)$ satisfying $U_{t}(t)=P(t) U(t)$, where $L(0)=$ $U(t)^{-1} L(t) U(t)$. In particular, returning to the case where $L(t)=\partial^{2} / \partial x^{2}+q(x, t)$, Lax showed that one can always find an odd-order differential expression $P_{2 n+1}(t)$ such that $\left[P_{2 n+1}(t), L(t)\right]$ is an operator of multiplication. The coefficients of $P_{2 n+1}(t)$ are then differential polynomials in $q(x, t)$, that is, polynomials of $q(x, t)$ and its $x$-derivatives. The commutator $\left[P_{2 n+1}, L\right]$ is then also a differential polynomial in $q$ and the evolution equation

$$
q_{t}=\left[P_{2 n+1}, L\right]
$$

defines the $n$-th equation of the KdV hierarchy.

More precisely, if $L=\partial^{2} / \partial x^{2}+q$, then any ordinary differential expression $P$, for which $[P, L]$ equals an operator of multiplication, is the sum of a polynomial $K \in \mathbb{C}[L]$ and an odd-order differential expression $P_{2 n+1}$, that is,

$$
P=K(L)+P_{2 n+1} .
$$

Upon rescaling the time variable, $P_{2 n+1}$ can be assumed to be monic. Moreover, choosing the polynomial $K$ appropriately, it may be shown that

$$
P_{2 n+1}=\sum_{j=0}^{n}\left[-\frac{1}{2} f_{j}^{\prime}+f_{j} \frac{d}{d x}\right] L^{n-j}
$$

for some integer $n \in \mathbb{N}_{0}(=\mathbb{N} \cup\{0\})$, where the functions $f_{j}$ satisfy the recursion relation

$$
\begin{aligned}
f_{0} & =1 \\
f_{j+1}^{\prime} & =\frac{1}{4} f_{j}^{\prime \prime \prime}+q f_{j}^{\prime}+\frac{1}{2} q^{\prime} f_{j}, \quad j=0, \ldots, n .
\end{aligned}
$$

Then

$$
[P, L]=\left[P_{2 n+1}, L\right]=\frac{1}{4} f_{n}^{\prime \prime \prime}+q f_{n}^{\prime}+\frac{1}{2} q^{\prime} f_{n}
$$

and introducing

$$
F_{n}(z, x, t)=\sum_{j=0}^{n} f_{n-j}(x, t) z^{j},
$$

this recursion relation becomes

$$
\left[P_{2 n+1}, L\right]=\frac{1}{2} F_{n}{ }^{\prime \prime \prime}+2(q-z) F_{n}{ }^{\prime}+q^{\prime} F_{n}
$$

Conversely, if $F_{n}(z, x)$ is a polynomial in $z$ such that $F_{n}{ }^{\prime \prime \prime}+4(q-z) F_{n}^{\prime}+2 q^{\prime} F_{n}$ does not depend on $z$, then its coefficients define a differential expression $P_{2 n+1}$ such that equation (2.3.4) is satisfied. In particular, if $F_{n}{ }^{\prime \prime \prime}+4(q-z) F_{n}^{\prime}+2 q^{\prime} F_{n}=0$, then $q$ is a stationary (i.e., $t$-independent) solution of one of the $n$-th equations in the KdV hierarchy. 
The first few equations of the KdV hierarchy explicitly read,

$$
\begin{aligned}
q_{t} & =q_{x}, \\
q_{t} & =\frac{1}{4} q_{x x x}+\frac{3}{2} q q_{x}+c_{1} q_{x}, \\
q_{t} & =\frac{1}{16} q_{x x x x x}+\frac{5}{8} q q_{x x x}+\frac{5}{4} q_{x} q_{x x}+\frac{15}{8} q^{2} q_{x}+c_{1}\left(\frac{1}{4} q_{x x x}+\frac{3}{2} q q_{x}\right)+c_{2} q_{x}, \\
& \text { etc. }
\end{aligned}
$$

where the $c_{\ell}$ denote integration constants encountered in solving the recursion (2.3.3) for $f_{j+1}$. Here we ought to mention that each of these equations is a completely integrable Hamiltonian system and that the sequence of these equations is intimately related to the sequence of conservation laws discovered by Gardner, Kruskal, and Miura [164].

However, the Lax method can be further generalized in a variety of ways: If one considers instead of $L$ a first-order $2 \times 2$-matrix differential expression (i.e., a Dirac-type operator), one arrives at the ZS hierarchy [242] which is associated with the nonlinear Schrödinger equation and, more generally, at the AKNS hierarchy [2] which includes the $\mathrm{KdV}$, the $\mathrm{mKdV}$, and the nonlinear Schrödinger hierarchies as special cases. If one considers instead of the second-order differential expression $L$ a differential expression of any order, one arrives at the Gelfand-Dickey hierarchy [44], [83]. Moreover, the formalism is not confined to differential expressions but extends to formal pseudo-differential expressions (including rational functions of $d / d x)$ needed in connection with the sine-Gordon and (modified) KadomtsevPetviashvili hierarchies.

2.4. The Algebra of Commuting Differential Expressions. As seen from (2.3.1), stationary (i.e., time-independent) Lax equations naturally lead to commuting differential expressions. Independently of this fact, the question of commuting differential expressions was raised by Floquet [69] in 1879. Some 25 years later, the question was again considered by Wallenberg [230] and Schur [192]. To this end, Schur developed the algebra of symbolic, that is, formal pseudo-differential expressions. He proves, in particular, the following statement: if $A$ and $B$ are two monic commuting differential expressions, then the coefficients of $A$ are differential polynomials in the coefficients of $B$ (for a contemporary approach to these results, see, e.g., Wilson [235]). The decisive step, however, was done by Burchnall and Chaundy in the 1920's. They proved the following result in [29].

Theorem 2.1. Let $A$ and $B$ be ordinary differential expressions of relatively prime orders $m$ and $n$, respectively. Then $[A, B]=0$ if and only if there exists a polynomial $f$ of the form

$$
f(\alpha, \beta)=\alpha^{n}-\beta^{m}+\sum_{\substack{j, k>0 \\ m j+n k<m n}} c_{j, k} \alpha^{j} \beta^{k},
$$

such that $f(A, B)=0$.

In this context, $f$ is called the Burchnall-Chaundy polynomial of $A$ and $B$. In [30] Burchnall and Chaundy constructed differential expressions $A$ and $B$ satisfying the equation $f(A, B)=0$ for a given polynomial $f$ of the form (2.4.1) (cf. also Baker [15], Burchnall and Chaundy [31]). More recent treatments of the BurchnallChaundy theory can be found, for instance, in Carlson and Goodearl [36], Gatto and Greco [81], Giertz, Kwong, and Zettl [101], Greco and Previato [103], Krichever [132], [133], Mumford [167], Previato [185], and Wilson [236]. 
Finally, we briefly return to the Lax pairs $(P, L)$ discussed in Section 2.3. Because of the Burchnall-Chaundy relationship we call $L$ (or the set of its coefficients) algebro-geometric if there exists a corresponding $P$ such that $[P, L]=0$. We will provide more precise definitions in the contexts of the KdV and the AKNS hierarchies in Definitions 3.1 and 4.2, respectively.

2.5. Elliptic Functions in a Nutshell. Elliptic functions provide some of the most important examples of algebro-geometric potentials. We present here a very brief account of Weierstrass' point of view. For general references see, for instance, Akhiezer [7], Chandrasekharan [37], Markushevich [153], and Whittaker and Watson [234].

A function $f: \mathbb{C} \rightarrow \mathbb{C}_{\infty}(=\mathbb{C} \cup\{\infty\})$ with two periods $a$ and $b$, the ratio of which is not real, is called doubly periodic. If all its periods are of the form $m_{1} a+m_{2} b$ where $m_{1}$ and $m_{2}$ are integers, then $a$ and $b$ are called fundamental periods of $f$.

A doubly periodic meromorphic function is called elliptic.

It is customary to denote the fundamental periods of an elliptic function by $2 \omega_{1}$ and $2 \omega_{3}$ with $\operatorname{Im}\left(\omega_{3} / \omega_{1}\right)>0$. We also introduce $\omega_{2}=\omega_{1}+\omega_{3}$ and $\omega_{4}=0$. The numbers $\omega_{1}, \ldots, \omega_{4}$ are called half-periods. The fundamental period parallelogram (f.p.p.) $\Delta$ is the half-open region consisting of the line segments $\left[0,2 \omega_{1}\right),\left[0,2 \omega_{3}\right)$ and the interior of the parallelogram with vertices $0,2 \omega_{1}, 2 \omega_{2}$ and $2 \omega_{3}$.

The class of elliptic functions with fundamental periods $2 \omega_{1}, 2 \omega_{3}$ is closed under addition, subtraction, multiplication, division by non-zero divisors and differentiation. If $f$ is an entire elliptic function, then $f$ is constant. A non-constant elliptic function $f$ must have at least one pole in $\Delta$ and the total number of poles in $\Delta$ is finite. The total number of poles of an elliptic function $f$ in $\Delta$ (counting multiplicities) is called the order of $f$. The sum of residues of an elliptic function $f$ at all its poles in $\Delta$ equals zero. In particular, the order of a non-constant elliptic function $f$ is at least 2 . The total number of points in $\Delta$ where the non-constant elliptic function $f$ assumes the value $A$ (counting multiplicities), denoted by $n(A)$, is equal to the order of $f$. In particular, $n(A) \geq 2$. Furthermore, $s(A)$, the sum of all the points in $\Delta$ where the non-constant elliptic function $f$ assumes the value $A$, is congruent to $s(\infty)$, the sum of all the points in $\Delta$ where $f$ has a pole, that is, $s(A)=s(\infty)+2 m_{1} \omega_{1}+2 m_{3} \omega_{3}$, where $m_{1}$ and $m_{3}$ are certain integers.

The function

$$
\wp\left(z ; \omega_{1}, \omega_{3}\right)=\frac{1}{z^{2}}+\sum_{\substack{m, n \in \mathbb{Z} \\(m, n) \neq(0,0)}}\left(\frac{1}{\left(z-2 m \omega_{1}-2 n \omega_{3}\right)^{2}}-\frac{1}{\left(2 m \omega_{1}+2 n \omega_{3}\right)^{2}}\right),
$$

or $\wp(z)$ for short, was introduced by Weierstrass. It is an even elliptic function of order 2 with fundamental periods $2 \omega_{1}$ and $2 \omega_{3}$. Its derivative $\wp^{\prime}$ is an odd elliptic function of order 3 with fundamental periods $2 \omega_{1}$ and $2 \omega_{3}$. Every elliptic function may be written as $R_{1}(\wp(z))+R_{2}(\wp(z)) \wp^{\prime}(z)$ where $R_{1}$ and $R_{2}$ are rational functions of $\wp$.

The numbers

$$
\begin{aligned}
& g_{2}=60 \sum_{\substack{m, n \in \mathbb{Z} \\
(m, n) \neq(0,0)}} \frac{1}{\left(2 m \omega_{1}+2 n \omega_{3}\right)^{4}}, \\
& g_{3}=140 \sum_{\substack{m, n \in \mathbb{Z} \\
(m, n) \neq(0,0)}} \frac{1}{\left(2 m \omega_{1}+2 n \omega_{3}\right)^{6}}
\end{aligned}
$$


are called the invariants of $\wp$. Since the coefficients of the Laurent expansions of $\wp(z)$ and $\wp^{\prime}(z)$ at $z=0$ are polynomials of $g_{2}$ and $g_{3}$ with rational coefficients, the function $\wp\left(z ; \omega_{1}, \omega_{3}\right)$ is also uniquely characterized by its invariants $g_{2}$ and $g_{3}$. One frequently also uses the notation $\wp\left(z \mid g_{2}, g_{3}\right)$.

The function $\wp(z)$ satisfies the first-order differential equation

$$
\wp^{\prime}(z)^{2}=4 \wp(z)^{3}-g_{2} \wp(z)-g_{3}
$$

and hence the equations

$$
\wp^{\prime \prime}(z)=6 \wp(z)^{2}-g_{2} / 2 \text { and } \wp^{\prime \prime \prime}(z)=12 \wp^{\prime}(z) \wp(z)
$$

which show that $-2 \wp$ is a stationary solution of the KdV equation (2.1.1).

The function $\wp^{\prime}$, being of order 3 , has three zeros in $\Delta$. Since $\wp^{\prime}$ is odd and elliptic it is obvious that these zeros are the half-periods $\omega_{1}, \omega_{2}=\omega_{1}+\omega_{3}$ and $\omega_{3}$. Let $e_{j}=\wp\left(\omega_{j}\right), j=1,2,3$. Then (2.5.1) implies that $4 e_{j}^{3}-g_{2} e_{j}-g_{3}=0$ for $j=1,2,3$. Therefore

$$
\begin{aligned}
0 & =e_{1}+e_{2}+e_{3}, \\
g_{2} & =-4\left(e_{1} e_{2}+e_{1} e_{3}+e_{2} e_{3}\right)=2\left(e_{1}^{2}+e_{2}^{2}+e_{3}^{2}\right), \\
g_{3} & =4 e_{1} e_{2} e_{3}=\frac{4}{3}\left(e_{1}^{3}+e_{2}^{3}+e_{3}^{3}\right) .
\end{aligned}
$$

Weierstrass also introduced two other functions denoted by $\zeta$ and $\sigma$. The Weierstrass $\zeta$-function is defined by

$$
\frac{d}{d z} \zeta(z)=-\wp(z), \quad \lim _{z \rightarrow 0}\left(\zeta(z)-\frac{1}{z}\right)=0 .
$$

It is a meromorphic function with simple poles at $2 m \omega_{1}+2 n \omega_{3}, m, n \in \mathbb{Z}$ having residues 1 . It is not periodic but quasi-periodic in the sense that

$$
\zeta\left(z+2 \omega_{j}\right)=\zeta(z)+2 \eta_{j}, \quad j=1,2,3,4,
$$

where $\eta_{j}=\zeta\left(\omega_{j}\right)$ for $j=1,2,3$ and $\eta_{4}=0$.

The Weierstrass $\sigma$-function is defined by

$$
\frac{\sigma^{\prime}(z)}{\sigma(z)}=\zeta(z), \quad \lim _{z \rightarrow 0} \frac{\sigma(z)}{z}=1 .
$$

$\sigma$ is an entire function with simple zeros at the points $2 m \omega_{1}+2 n \omega_{3}, m, n \in \mathbb{Z}$. Under translation by a period $\sigma$ behaves according to

$$
\sigma\left(z+2 \omega_{j}\right)=-\sigma(z) e^{2 \eta_{j}\left(z+\omega_{j}\right)}, \quad j=1,2,3 .
$$

Next we recall the following fundamental theorems.

Theorem 2.2. Given an elliptic function $f$ with fundamental periods $2 \omega_{1}$ and $2 \omega_{3}$, let $b_{1}, \ldots, b_{r}$ be the distinct poles of $f$ in $\Delta$. Suppose the principal part of the Laurent expansion near $b_{k}$ is given by

$$
\sum_{j=1}^{\beta_{k}} \frac{A_{j, k}}{\left(z-b_{k}\right)^{j}}, \quad k=1, \ldots, r .
$$

Then

$$
f(z)=C+\sum_{k=1}^{r} \sum_{j=1}^{\beta_{k}}(-1)^{j-1} \frac{A_{j, k}}{(j-1) !} \zeta^{(j-1)}\left(z-b_{k}\right),
$$


where $C$ is a suitable constant and $\zeta$ is constructed from the fundamental periods $2 \omega_{1}$ and $2 \omega_{3}$. Conversely, every such function is an elliptic function if $\sum_{k=1}^{r} A_{1, k}=0$.

Theorem 2.3. Given an elliptic function $f$ of order $n$ with fundamental periods $2 \omega_{1}$ and $2 \omega_{3}$, let $a_{1}, \ldots, a_{n}$ and $b_{1}, \ldots, b_{n}$ be the zeros and poles of $f$ in $\Delta$ repeated according to their multiplicities. Then

$$
f(z)=C \frac{\sigma\left(z-a_{1}\right) \cdots \sigma\left(z-a_{n}\right)}{\sigma\left(z-b_{1}\right) \cdots \sigma\left(z-b_{n-1}\right) \sigma\left(z-b_{n}^{\prime}\right)},
$$

where $C$ is a suitable constant, $\sigma$ is constructed from the fundamental periods $2 \omega_{1}$ and $2 \omega_{3}$ and

$$
b_{n}^{\prime}-b_{n}=\left(a_{1}+\ldots+a_{n}\right)-\left(b_{1}+\ldots+b_{n}\right)
$$

is a period of $f$. Conversely, every such function is an elliptic function.

Finally, we turn to elliptic functions of the second kind, the central object in our analysis. A meromorphic function $\psi: \mathbb{C} \rightarrow \mathbb{C} \cup\{\infty\}$ for which there exist two complex constants $\omega_{1}$ and $\omega_{3}$ with non-real ratio and two complex constants $\rho_{1}$ and $\rho_{3}$ such that for $i=1,3$

$$
\psi\left(z+2 \omega_{i}\right)=\rho_{i} \psi(z)
$$

is called elliptic of the second kind. We call $2 \omega_{1}$ and $2 \omega_{3}$ the quasi-periods of $\psi$. Together with $2 \omega_{1}$ and $2 \omega_{3}, 2 m_{1} \omega_{1}+2 m_{3} \omega_{3}$ are also quasi-periods of $\psi$ if $m_{1}$ and $m_{3}$ are integers. If every quasi-period of $\psi$ can be written as an integer linear combination of $2 \omega_{1}$ and $2 \omega_{3}$, then these are called fundamental quasi-periods.

Theorem 2.4. A function $\psi$ which is elliptic of the second kind and has fundamental quasi-periods $2 \omega_{1}$ and $2 \omega_{3}$ can always be put in the form

$$
\psi(z)=C \exp (\lambda z) \frac{\sigma\left(z-a_{1}\right) \cdots \sigma\left(z-a_{n}\right)}{\sigma\left(z-b_{1}\right) \cdots \sigma\left(z-b_{n}\right)}
$$

for suitable constants $C, \lambda, a_{1}, \ldots, a_{n}$ and $b_{1}, \ldots, b_{n}$. Here $\sigma$ is constructed from the fundamental periods $2 \omega_{1}$ and $2 \omega_{3}$. Conversely, every such function is elliptic of the second kind.

Theorem 2.5. Given numbers $\alpha_{1}, \ldots, \alpha_{m}$ and $\beta_{1}, \ldots, \beta_{m}$ such that $\beta_{k} \neq \beta_{\ell}(\bmod \Delta)$ for $k \neq \ell$, the following identity holds

$$
\prod_{j=1}^{m} \frac{\sigma\left(x-\alpha_{j}\right)}{\sigma\left(x-\beta_{j}\right)}=\sum_{j=1}^{m} \frac{\prod_{k=1}^{m} \sigma\left(\beta_{j}-\alpha_{k}\right)}{\prod_{\ell=1, \ell \neq j}^{m} \sigma\left(\beta_{j}-\beta_{\ell}\right)} \frac{\sigma\left(x-\beta_{j}+\beta-\alpha\right)}{\sigma\left(x-\beta_{j}\right) \sigma(\beta-\alpha)},
$$

where

$$
\alpha=\sum_{j=1}^{m} \alpha_{j} \text { and } \beta=\sum_{j=1}^{m} \beta_{j}
$$

and $\sigma$ is constructed from the fundamental periods $2 \omega_{1}$ and $2 \omega_{3}$.

Sketch of proof. Since this result seems less familiar than those above we will briefly sketch its proof. Denote the left- and right-hand sides of (2.5.2) by $f$ and $g$, respectively. Both $f$ and $g$ are elliptic functions of the second kind associated with the same Floquet multipliers (with respect to translations by $2 \omega_{k}, k=1,3$ ). Their quotient is therefore an elliptic function. Also $f$ and $g$ have the same poles and zeros 
taking multiplicities into account. The statement about the zeros is a consequence of the identity (cf. [234], p. 451)

$$
\sum_{j=1}^{m}\left(\frac{\prod_{k=1}^{m} \sigma\left(\gamma_{j}-\delta_{k}\right)}{\prod_{\ell=1, \ell \neq j}^{m} \sigma\left(\gamma_{j}-\gamma_{\ell}\right)}\right)=0 \text { if } \sum_{j=1}^{m} \gamma_{j}=\sum_{j=1}^{m} \delta_{j} .
$$

Therefore $f / g$ is entire and hence constant. Since all residues are equal to one, $f=g$.

2.6. Hill's Equation and Its Spectral Theory. The study of linear homogeneous differential equations with periodic coefficients predates the late nineteenth century, but the equation $(L y)(x)=y^{\prime \prime}(x)+q(x) y(x)=z y(x)$, where $q(x)$ is a continuous, real-valued, periodic function of a real variable $x$ and $z$ is a real parameter, has generally been called Hill's equation since its appearance in the study of the lunar perigee by Hill [113] in 1877. In addition to its applications in celestial mechanics, this equation has found countless applications in quantum mechanics, where it becomes Schrödinger's equation and is used, for instance, to model crystal structures of solids.

Periodic differential equations are usually studied by applying Floquet theory. Floquet theory (first developed by Floquet [70], [71] starting in 1880) specifies the general structure of solutions of systems of periodic differential equations. Consider the equation $\underline{y}^{\prime}(x)=Q(x) \underline{y}(x)$, where $Q$ is an $n \times n$ matrix whose entries are continuous (for simplicity) and periodic with period $\Omega>0$ and $\underline{y}(x)$ is $\mathbb{C}^{n}$-valued. Let $\mathcal{Y}$ be the space of solutions of $\underline{y}^{\prime}(x)=Q(x) \underline{y}(x)$ and $\mathcal{T}_{\Omega}$ the restriction of $\underline{y} \mapsto \underline{y}(\cdot+\Omega)$ to $\mathcal{Y}$. Floquet theory then amounts to the study of the operator $\mathcal{T}_{\Omega}$. Since $\mathcal{T}_{\Omega}$ maps the $n$-dimensional vector space $\mathcal{Y}$ to itself, the problem is reduced to a problem in linear algebra. The eigenvalues and eigenfunctions of $\mathcal{T}_{\Omega}$ are called Floquet multipliers and Floquet functions, respectively. For general references on Floquet theory see, for instance, Arscott [10]; Coddington and Levinson [42], Ch. 3; Eastham [57]; Ince [118], Sect. 10.8; Magnus and Winkler [150]; Marchenko [152], Sect. 3.4; McKean and van Moerbeke [159]; and Yakubovich and Starzhinskii [239]. In the special case of Hill's equation $(L y)(x)=y^{\prime \prime}(x)+q(x) y(x)=z y(x)$, we will denote the translation operator restricted to the set of solutions $\left(y(z, x), y^{\prime}(z, x)\right)^{t} \in$ $\mathcal{Y}(z)$ of the associated first-order system by $\mathcal{T}_{\Omega}(z)$.

Several differential operators (resp., boundary value problems) are studied in connection with Hill's equation,

$$
(L y)(x)=y^{\prime \prime}(x)+q(x) y(x)=z y(x), \quad q(x+\Omega)=q(x) .
$$

(1) The maximally defined operator $H$ in $L^{2}(\mathbb{R})$ associated with $L$.

(2) Auxiliary operators in $L^{2}\left(\left[x_{0}, x_{0}+\Omega\right]\right)$ associated with $L$ and certain families of boundary conditions, in particular, Dirichlet boundary conditions.

(3) The operators in $L^{2}\left(\left[x_{0}, x_{0}+\Omega\right]\right)$ associated with $L$ and cyclic boundary conditions $y\left(x_{0}+\Omega\right)=\exp (i \theta) y\left(x_{0}\right), y^{\prime}\left(x_{0}+\Omega\right)=\exp (i \theta) y^{\prime}\left(x_{0}\right), \theta \in[0,2 \pi)$, in particular, periodic $(\theta=0)$ and anti-periodic $(\theta=\pi)$ boundary conditions.

Floquet theory provides us with a handle on the problem of determining spectral properties of these operators: the periodic eigenvalues are given as the (necessarily real) zeros of $\operatorname{tr}\left(\mathcal{T}_{\Omega}(z)\right)-2$, while the anti-periodic eigenvalues are given as the (necessarily real) zeros of $\operatorname{tr}\left(\mathcal{T}_{\Omega}(z)\right)+2$, and the spectrum of $H$ coincides with the conditional stability set $\mathcal{S}(q)$, that is, the set of all values $z \in \mathbb{R}$ such that $(L y)(x)=z y(x)$ has a nontrivial bounded solution with respect to $x \in \mathbb{R}$. This was 
first shown by Wintner [237], [238] in 1947/48. The conditional stability set, in turn, may be characterized as the set of all $z \in \mathbb{R}$ such that $L y=z y$ has a Floquet multiplier of absolute value one. Since

$$
\operatorname{det}\left(\mathcal{T}_{\Omega}(z)\right)=1
$$

the Floquet multipliers, being the eigenvalues of $\mathcal{T}_{\Omega}(z)$, are given as the zeros of $\rho^{2}-\rho \operatorname{tr}\left(\mathcal{T}_{\Omega}(z)\right)+1$ and we get

$$
\mathcal{S}(q)=\left\{z \in \mathbb{R} \mid-2 \leq \operatorname{tr}\left(\mathcal{T}_{\Omega}(z)\right) \leq 2\right\} .
$$

The conditional stability set consists of countably (possibly finitely) many closed intervals (plus possibly a half-line), whose endpoints coincide with points where only one (linearly independent) Floquet solution exists (which is necessarily (anti-)periodic). This was first shown by Hamel [109] in 1913 (see also Liapunov [149], who treated the case $y^{\prime \prime}=\lambda p y$ with a periodic function $p$ in 1899, and Haupt [110], who corrected a mistake in Hamel's paper). Hence the (anti-)periodic eigenvalues determine the spectrum of $H$. From the periodic and anti-periodic eigenvalues repeated according to their multiplicity and ordered as a decreasing sequence denoted by $E_{0}, E_{1}, \ldots$ (observing that for normal operators the algebraic and geometric multiplicities of eigenvalues coincide), one infers

$$
\sigma(H)=\bigcup_{j=0}^{\infty}\left[E_{2 j+1}, E_{2 j}\right] .
$$

Typically, the spectral bands $\left[E_{2 j+1}, E_{2 j}\right]$ are separated by spectral gaps $\left(E_{2 j+2}\right.$, $\left.E_{2 j+1}\right)$. However, since the (anti-)periodic eigenvalues may be twofold degenerate, some gaps (or even all gaps) may close. If only finitely many gaps are present, one calls $q$, the potential coefficient in $L$, a finite-gap, or a finite-band potential.

A trivial example of a finite-band potential is of course the constant potential $q(x)=c$. The first in this century to discuss a nontrivial example was Ince [117] around 1940, who treated in depth Lamé's potential

$$
q(x)=-s(s+1) \wp\left(x+\omega_{3}\right), \quad s \in \mathbb{N},
$$

with fundamental half periods $\omega_{1} \in \mathbb{R}$ and $\omega_{3} \in i \mathbb{R}$. Under these conditions $q$ is real-valued and real-analytic in $x$. Ince showed that for all but $2 n+1$ values of $z \in \mathbb{R}$, the equation $y^{\prime \prime}(x)+q(x) y(x)=z y(x)$ has two linearly independent (anti-)periodic eigenvalues; that is, $q$ in (2.6.1) is a finite-band potential (see also Akhiezer [6], Erdelyi [65], Turbiner [227], and Ward [231]). However, a closer look at the classical works of Hermite [112] and Halphen [108] (see also Klein [124]) on Lamé's equation at the end of the last century reveals that these results were actually well-known (but not yet put in a Floquet-theoretic language) as can be inferred, for instance, from Whittaker-Watson's treatise [234], Sect. 23.7.

In 1909 Birkhoff [22] compared eigenvalues of various boundary value problems associated with $L$ on a finite interval. His results show that there is precisely one Dirichlet eigenvalue in each of the intervals $\left[E_{2 j+2}, E_{2 j+1}\right]$ (i.e., in the closure of the gaps). In contrast to the (anti-)periodic eigenvalues, the Dirichlet eigenvalues associated with the interval $\left[x_{0}, x_{0}+\Omega\right]$ vary with $x_{0}$ in $\left[E_{2 j+2}, E_{2 j+1}\right]$ if $E_{2 j+2}<$ $E_{2 j+1}$. Writing $\mathbb{R}=\cup_{n \in \mathbb{Z}}\left[x_{0}+n \Omega, x_{0}+(n+1) \Omega\right]$, one finds that a comparison of the Dirichlet problems on $\left[x_{0}, x_{0}+\Omega\right]$ and on $\left(-\infty, x_{0}\right) \cup\left(x_{0}, \infty\right)$ assuming $q(x)$ to be $\Omega$ periodic yields the same Dirichlet spectra in the spectral gaps $\left(E_{2 j+2}, E_{2 j+1}\right)$ with $E_{2 j+2}<E_{2 j+1}$. Explicit formulas for the monotone rate of change of various kinds 
of eigenvalues (including Dirichlet eigenvalues) with respect to varying $x_{0} \in \mathbb{R}$ can be found in Kong and Zettl [125], [126] and the literature therein. In [88] this phenomenon is related to Green's functions and rank-one perturbations of resolvents.

If $q$ is a locally integrable, real-valued, periodic function with period $\Omega>0$, we have equivalence of the following statements:

(A) $q$ is a finite-band potential.

(B) For only finitely many values of $z$ the differential equation $y^{\prime \prime}(x)+q(x) y(x)=$ $z y(x)$ fails to have two linearly independent Floquet solutions.

(C) The Dirichlet boundary value problem on the interval $\left[x_{0}, x_{0}+\Omega\right]$ has only a finite number of eigenvalues depending on $x_{0}$.

Spectral theoretic aspects of complex-valued periodic potentials $q$ have been investigated by Rofe-Beketov [187] and Tkachenko [210] (see also Birnir [23], [24], Kotani [130], McGarvey [156], [157], [158], Sansuc and Tkachenko [189], [190], [191], and Tkachenko [211], [212], [214] for recent results). They found that the spectrum of $H$ and the conditional stability set of $L$ still coincide, that is,

$$
\mathcal{S}(q)=\left\{z \in \mathbb{C} \mid-2 \leq \operatorname{tr}\left(\mathcal{T}_{\Omega}(z)\right) \leq 2\right\} .
$$

Since the conditional stability set is given as the preimage of $[-2,2]$ under an entire function, it turns out that the spectrum of $H$ consists of countably many (possibly finitely many) regular analytic arcs. While the term "finite-gap potential" is now rendered meaningless, it still makes perfect sense to call a potential a finite-band potential if the spectrum is a finite union of regular analytic arcs. In contrast to the real-valued case, these spectral arcs can now cross each other; see, for instance, [92], [175] for explicit examples exhibiting this phenomenon.

Returning to the real-valued case, the isospectral set $I\left(q_{0}\right)$ of a given periodic potential $q_{0} \in C(\mathbb{R})$ with period $\Omega>0$ (i.e., the set of all $\Omega$-periodic $q \in C(\mathbb{R})$ whose $2 \Omega$-periodic eigenvalues coincide with that of $q_{0}$ ) turns out to be a manifold, in fact, a (generally infinite dimensional) torus generated by a product of circles. Each circle is uniquely associated with a spectral gap $\left(E_{2 j+2}, E_{2 j+1}\right), E_{2 j+2}<E_{2 j+1}$ and the periodic motion of the Dirichlet eigenvalue in the gap $\left(E_{2 j+2}, E_{2 j+1}\right)$ corresponding to the interval $[x, x+\Omega]$ as a function of $x \in \mathbb{R}$. In the special case where $q$ is realvalued and periodic, and the $L^{2}$-spectrum of $-d^{2} / d x^{2}+q(x)$ is a half-line $\left(-\infty, E_{0}\right]$, Borg [26] proved the celebrated inverse spectral result $q(x)=E_{0}$ for all $x \in \mathbb{R}$. A quick proof of this uniqueness result and extensions to real-valued reflectionless potentials $q$ with associated half-line spectrum $\left(-\infty, E_{0}\right]$ follows from the trace formula proved in [88] (observing $\xi(\lambda, x)=1 / 2$ for $\lambda<E_{0}$ in the corresponding trace formula (3.1) for $q(x)$ in [88]). Similarly, if $q$ is real-valued and periodic, and the $L^{2}$-spectrum of $-d^{2} / d x^{2}+q(x)$ is of the type $\left(-\infty, E_{2}\right] \cup\left[E_{1}, E_{0}\right], E_{2}<E_{1}$, Hochstadt [115] proved that $q(x)=-2 \wp\left(x+\omega_{3}+\alpha\right)$, that is, the Lamé potential (2.6.1) for $n=1$, where $\wp(x)$ denotes the Weierstrass elliptic function associated with some period lattice $\omega_{1}>0,-i \omega_{3}>0$ (depending on $E_{0}, E_{1}$, and $E_{2}$ ) and $\alpha \in \mathbb{R}$. The isospectral torus for real-valued periodic potentials with three or more spectral bands is described, for instance, in Buys and Finkel [33], Finkel, Isaacson, and Trubowitz [67], Gesztesy, Simon, and Teschl [89], Gesztesy and Weikard [91], Iwasaki [123], McKean van Moerbeke [159], and McKean and Trubowitz [160].

It should be emphasized in this context that the assumption of real-valuedness of $q(x)$ cannot be dropped as shown by the well-known example $q(x)=\exp (i x)$, 
with associated $L^{2}$-spectrum $(-\infty, 0]$ (see the paragraph following Remark 3.5 for more details).

2.7. Periodic KdV Potentials. In 1974, Novikov [168] investigated the Cauchy problem of the KdV equation in the case of periodic initial data. He noted that the right generalization of multi-soliton solutions, which are stationary solutions of appropriate higher-order KdV equations, are the finite-band potentials; that is, he proved the following result.

Theorem 2.6. If $q$ is a real-valued, periodic, stationary solution of an $n$-th order $K d V$ equation, then the $L^{2}(\mathbb{R})$-spectrum associated with $d^{2} / d x^{2}+q(x)$ has at most $n$ finite bands.

Within a year, Dubrovin [52] and Flaschka [68] also proved the converse, and Dubrovin and Novikov [56] used these results to solve the Cauchy problem of the $\mathrm{KdV}$ equation in the case of finite-band initial data. Hence the statement,

(D) $q$ is a stationary solution of a higher-order $\mathrm{KdV}$ equation,

is equivalent to any of the statements $(\mathrm{A})-(\mathrm{C})$ in Section 2.6 for real-valued $q$.

Moreover, it became clear from these investigations that generically, finite-band potentials will only be quasi-periodic with respect to $x$ and not periodic in $x$.

About the same time, Its and Matveev [122] derived their celebrated formula for finite-gap solutions $q(x, t)$ in terms of the Riemann theta function associated with the underlying hyperelliptic curve and a fixed homology basis on it (cf. [16], Ch. 3; [86], Ch. 1; [169], Ch. II). Subsequent extensions of this formula to general (matrix-valued) integrable systems were developed by Dubrovin [53], [54], [55] and Krichever [132], [133], [136]. A new approach to finite-band solutions of the KdV hierarchy in terms of Kleinian functions was recently developed by Buchstaber, Enol'skii, and Leykin [27], [28].

2.8. Elliptic KdV Potentials. While the considerations of the preceding subsections pertain to general solutions of the stationary KdV hierarchy, we now concentrate on the additional restriction that $q$ be an elliptic function and hence return to our main subject, elliptic finite-band potentials $q$ for $L=d^{2} / d x^{2}+q(x)$, or, equivalently, elliptic solutions of the stationary KdV hierarchy. The remarkable finite-gap example of the Lamé potentials (2.6.1) due to Hermite [112] and Halphen [108] in the last century, brought back into the limelight by Ince [117] around 1940, remained the only explicit elliptic finite-gap example until the $\mathrm{KdV}$ flow $q_{t}=\frac{1}{4} q_{x x x}+\frac{3}{2} q q_{x}$, with initial condition $q(x, 0)=-6 \wp\left(x+\omega_{3}\right)$, was explicitly integrated by Dubrovin and Novikov [56] in 1975 (see also Enol'skii [60], [61], [62], Its and Enol'skii [121]) and found to be of the type

$$
q(x, t)=-2 \sum_{j=1}^{3} \wp\left(x-x_{j}(t)\right)
$$

for appropriate $\left\{x_{j}(t)\right\}_{1 \leq j \leq 3}$. Due to the unitary evolution operator $U_{n}(t)$ constructed with the help of $P_{2 n+1}(t)$ via $U_{n, t}(t)=P_{2 n+1}(t) U_{n}(t)$, all potentials $q(x, t)$ in $(2.8 .1)$ are isospectral to $q(x, 0)=-6 \wp\left(x+\omega_{3}\right)$.

In 1977, Airault, McKean and Moser, in their seminal paper [5], presented the first systematic study of the isospectral torus $I_{\mathbb{R}}\left(q_{0}\right)$ of real-valued smooth potentials 
$q_{0}(x)$ of the type

$$
q_{0}(x)=-2 \sum_{j=1}^{M} \wp\left(x-x_{j}\right)
$$

with a finite-gap spectrum. Among a variety of results they proved that any element $q$ of $I_{\mathbb{R}}\left(q_{0}\right)$ is an elliptic function of the type (2.8.2) (with different $x_{j}$ ), with $M$ constant throughout $I_{\mathbb{R}}\left(q_{0}\right)$ and $\operatorname{dim} I_{\mathbb{R}}\left(q_{0}\right) \leq M$. In particular, if $q_{0}$ evolves according to any equation of the KdV hierarchy, it remains an elliptic finite-gap potential. The potential (2.8.2) is intimately connected with completely integrable many-body systems of the Calogero-Moser-type [34], [165] (see also Bennequin [21], Birnir [25], Calogero [35], Chudnovsky and Chudnovsky [38], [40], Olshanetsky and Perelomov [170], and Ruijsenaars [188]). This connection with integrable particle systems was subsequently exploited by Krichever [135] in his construction of elliptic algebro-geometric solutions of the Kadomtsev-Petviashvili equation. In the KdV context of (2.8.2), Krichever's approach relies on the ansatz

$$
\psi_{0}(z, x)=e^{\kappa(z) x} \sum_{j=1}^{M} A_{j}(z) \Phi\left(x-x_{j}, \rho(z)\right),
$$

for the Floquet solutions of $L_{0}=d^{2} / d x^{2}+q_{0}(x)$, where

$$
\Phi(x, \rho)=\frac{\sigma(x-\rho)}{\sigma(x) \sigma(-\rho)} e^{\zeta(\rho) x}
$$

(assuming for simplicity the generic case $x_{j} \neq x_{k}(\bmod \Delta)$ for $j \neq k$ ). Applying $L_{0}$ to (2.8.3) then yields an M-sheeted covering of the torus associated with the fundamental periods $2 \omega_{1}, 2 \omega_{3}$ and hence a description of the underlying algebraic curve. (We will briefly comment on this ansatz in Remark 3.9.)

The next breakthrough occurred in 1988 when Verdier [229] published new explicit examples of elliptic finite-gap potentials. Verdier's examples spurred a flurry of activities and inspired Belokolos and Enol'skii [18], [19], Smirnov [195], and subsequently Taimanov [207] and Kostov and Enol'skii [129] to find further such examples by combining the reduction process of Abelian integrals to elliptic integrals (see Babich, Bobenko and Matveev [13], [14]; Belokolos, Bobenko, Enol'skii, Its, and Matveev [16], Ch. 7; and Belokolos, Bobenko, Mateev, and Enol'skii [17]) with the aforementioned techniques of Krichever [135], [136]. This development finally culminated in a series of recent results of Treibich and Verdier [222], [223], [224] where it was shown that a general complex-valued potential of the form

$$
q(x)=-\sum_{j=1}^{4} d_{j} \wp\left(x-\omega_{j}\right)
$$

$\left(\omega_{2}=\omega_{1}+\omega_{3}, \omega_{4}=0\right)$ is a finite-gap potential if and only if $d_{j} / 2$ are triangular numbers, that is, if and only if

$$
d_{j}=s_{j}\left(s_{j}+1\right) \text { for some } s_{j} \in \mathbb{Z}, 1 \leq j \leq 4 .
$$

We shall from now on refer to potentials of the type

$$
q(x)=-\sum_{j=1}^{4} s_{j}\left(s_{j}+1\right) \wp\left(x-\omega_{j}\right), s_{j} \in \mathbb{Z}, 1 \leq j \leq 4
$$


as Treibich-Verdier potentials. The methods of Treibich and Verdier (see also Colombo, Pirola, and Previato [43], Previato [183], [184], Previato and Verdier [186]) are based on hyperelliptic tangent covers of the torus $\mathbb{C} / \Lambda(\Lambda$ being the period lattice generated by $2 \omega_{1}$ and $2 \omega_{3}$ ).

The state of the art of elliptic finite-gap solutions up to 1993 was recently reviewed in a special issue of Acta Applicandae Math.; see, for instance, Belokolos and Enol'skii [20], Enol'skii and Kostov [64], Krichever [138], Smirnov [197], Taimanov [208], and Treibich [220]. For more recent results see Eilbeck and Enol'skii [58], [59], [63] and Smirnov [198], [201]. Since a complete characterization of all elliptic finite-gap solutions of the stationary KdV hierarchy was still open at that time, we developed a new approach to this characterization problem to be described in Sections 3 and 4. As alluded to at the end of our introduction, Caloger-Moser-type models are again an intensive object of study.

Since we will also discuss stationary rational solutions of the KdV hierarchy in Section 3 we should mention the case where $\wp(x)$ in (2.8.2) degenerates into $x^{-2}$ as discussed, for instance, by Airault, McKean, and Moser [5], Krichever [134], [137], Moser [165], [166], Pelinovsky [178], and Shiota [194].

2.9. Linear Differential Equations in the Complex Domain. While all the developments described in previous subsections were in place around 1993, one final point, the connection of this subject to the classical area of differential equations in the complex domain, was made only around 1994 when we started to work on [97]. In the following we will remind the reader about this fundamental, but thus far missing, piece, which plays a decisive role in the remainder of this review.

In the late 1830's, Lamé studied Laplace's equation in confocal coordinates. After some appropriate changes of variables this led to the differential equation (2.6.1), its Weierstrass form. At the end of the last century, Lamé's equation (especially in Jacobi's form) was studied intensively by Hermite [111], who obtained the general solution of the equation for integer values of $n$ and any value of $z$. Picard proceeded to consider first general second-order equations [179], then $n$-th order equations [180] (see also Floquet [72], [73], [74], Mittag-Leffler [162], and Halphen [106], [107], [108]), and finally first-order systems [181] whose coefficients are elliptic functions. Consider the differential equation

$$
\underline{y}^{\prime}(x)=Q(x) \underline{y}(x),
$$

where $y(x)$ is $\mathbb{C}^{n}$-valued and $Q$ is an $n \times n$ matrix whose entries are elliptic functions with a common period lattice spanned by the fundamental periods $2 \omega_{1}$ and $2 \omega_{3}$. Let $\mathcal{Y}$ be the space of solutions of (2.9.1), and denote the restriction of the translation operator $\underline{y} \mapsto \underline{y}\left(\cdot+2 \omega_{j}\right)$ to $\mathcal{Y}$ by $\mathcal{T}_{j}$. Using this notation, Picard's theorem reads as follows (see also Akhiezer; [7], Sects. 58, 59; Burkardt [32], Ch. 15; Forsyth [75], Ch. IX; Gray [102], Sect. 6.1; Halphen [108], Ch. XIII; Ince [118], Sect. 15.6; Krause [131], Vol. 2, Ch. 3; and Picard [182], Sect. III.V).

Theorem 2.7. Assume that the first-order system (2.9.1) has a meromorphic fundamental system of solutions. Then there exists at least one solution $\psi$ which is elliptic of the second kind; that is, the components of $\psi$ are meromorphic and $\mathcal{T}_{j} \psi=\rho_{j} \psi$ for $j=1,3$ for suitable constants $\left.\rho_{1}, \rho_{3} \in \mathbb{C} \backslash \overline{\{0}\right\}$. If, in addition, one of the operators $\mathcal{T}_{1}$ and $\mathcal{T}_{3}$ has distinct eigenvalues, then there exists a fundamental system of solutions of (2.9.1) which are elliptic of the second kind. 
The explicit Floquet-type structure of solutions of (2.9.1) in terms of a doubly periodic vector, powers of $x$, powers of the Weierstrass zeta-function, and an exponential contribution has recently been determined in [90].

About the time Floquet, Fuchs, Hermite, Mittag-Leffler, and Picard (cf. the historical discussions in Gray [102], Ch. VI) developed the theory of differential equations with elliptic coefficients, Floquet [70], [71] also established his celebrated results for linear, homogeneous differential equations with simply-periodic coefficients. From a historical perspective it is perhaps interesting to note that Floquet assumed the coefficients, as well as the general solution of the equation, to be meromorphic in order to arrive at the existence of periodic solutions of the second kind; that is, he obtained the precise analog of Picard's result. Only later was it realized that his theorem extends to continuous periodic coefficients on $\mathbb{R}$ without any reference to meromorphic fundamental systems. The solutions Floquet called "periodic of the second kind" are today generally called Floquet solutions.

Next, we mention another theorem with a similar flavor that concerns differential equations with rational coefficients and meromorphic fundamental systems of solutions and hence is applicable to the study of rational algebro-geometric $\mathrm{KdV}$ potentials. In 1885, Halphen [107] published the following result.

Theorem 2.8. Assume that the differential equation $y^{(n)}(x)+q_{1}(x) y^{(n-1)}(x)+$ $\cdots+q_{n}(x) y(x)=0$ has rational coefficients bounded at infinity and a meromorphic fundamental system of solutions. Then the general solution is of the form $R_{1}(x) \exp \left(\lambda_{1} x\right)+\cdots+R_{n}(x) \exp \left(\lambda_{n} x\right)$, where $R_{1}, \ldots, R_{n}$ are rational functions.

It should be emphasized that the principal hypothesis in Theorems 2.7 and 2.8, the existence of a meromorphic fundamental system of solutions, can be verified in a straightforward manner by applying the Frobenius method (see, e.g., Coddington and Levinson [42], Sect. 4.8; Forsyth [75], Ch. III; Hille [114], Ch. 9; Ince [118], Ch. XVI; and Whittaker and Watson [234], Ch. X) to each pole of $q$ in the fundamental period parallelogram.

Finally we mention an observation made by Appell [9]. Let $y_{1}(x)$ and $y_{2}(x)$ be linearly independent solutions of $y^{\prime \prime}(x)+u(x) y(x)=0$. Then $y_{1}(x)^{2}, y_{1}(x) y_{2}(x)$, and $y_{2}(x)^{2}$ are linearly independent solutions of

$$
w^{\prime \prime \prime}(x)+4 u(x) w^{\prime}(x)+2 u^{\prime}(x) w(x)=0 .
$$

This equation is easily integrated and yields

$$
g^{\prime}(x)^{2}-2 g(x) g^{\prime \prime}(x)-4 u(x) g(x)^{2}=W\left(y_{1}, y_{2}\right)^{2},
$$

where $g(x)$ is the product of any two solutions $y_{1}(x)$ and $y_{2}(x)$ of $y^{\prime \prime}(x)+u(x) y(x)=$ 0 and $W\left(y_{1}, y_{2}\right)$ denotes their ( $x$-independent) Wronskian. This innocent looking fact will be of great importance in our analysis later. In fact, a comparison of equations (2.3.4) and (2.9.2) reveals another connection between the KdV hierarchy and the Schrödinger-type equation $y^{\prime \prime}(x)+u(x) y(x)=0$. Moreover, since the formal Green's function $G\left(z, x, x^{\prime}\right)$ of $d^{2} / d x^{2}+q(x)$ on the diagonal $x=x^{\prime}$ is of the type $y_{1}(z, x) y_{2}(z, x) / W\left(y_{1}(z), y_{2}(z)\right),(2.9 .3)$, with $u(x)=q(x)-z$, is equivalent to the well-known universal nonlinear second-order differential equation satisfied by $G(z, x, x)$ (see also Gelfand and Dickey, [82], [84]).

It should be noted that Drach [48], [49], [50] (see also [41]) used (2.9.2) to derive a class of completely integrable systems now known as the stationary KdV hierarchy as early as 1918/19. It appears he was the first to make the explicit connection 
between completely integrable systems and spectral theory. More than 55 years later, Gelfand and Dickey [82], [84] also based some of their celebrated work on the KdV hierarchy on (2.9.2).

\section{Algebro-geometric, and especially, elliptic KdV potentials}

Definition 3.1. Suppose $q$ is meromorphic and let $L$ be the differential expression $L=d^{2} / d x^{2}+q(x)$. Then $q$ is called an algebro-geometric KdV potential (or simply algebro-geometric) if $q$ is a solution of some equation of the stationary $\mathrm{KdV}$ hierarchy.

Equivalently, we could define the meromorphic function $q$ as algebro-geometric if any one of the following three conditions is satisfied.

(1) There exists an odd-order differential expression $P$ such that $[P, L]=0$ (according to the result of Burchnall and Chaundy).

(2) There exists an ordinary differential expression $P$ of odd order and a polynomial $R$ such that $P^{2}=R(L)$.

(3) There exists a function $F: \mathbb{C}^{2} \rightarrow \mathbb{C}_{\infty}$, which is a polynomial in the first variable, meromorphic in the second, and which satisfies

$$
F^{\prime \prime \prime}(z, x)+4(q(x)-z) F^{\prime}(z, x)+2 q^{\prime}(x) F(z, x)=0
$$

(cf. equation (2.3.4)).

It can be shown (see Theorem 6.10 by Segal and Wilson [193]) that any solution of any of the stationary KdV equations is necessarily meromorphic. Hence the assumption that $q$ is meromorphic is actually redundant in Definition 3.1.

3.1. Periodic KdV Potentials. If $q$ is real-valued, locally integrable, and periodic, we obtain the equivalence of the following statements from the works of Birkhoff and Hamel described in Section 2.6.

(i) $q$ is algebro-geometric with $P^{2}=\prod_{j=0}^{2 n}\left(L-\lambda_{j}\right)$.

(ii) $q$ is finite-band with spectrum the union of $\left(-\infty, \lambda_{2 n}\right]$ and $n$ compact bands $\left[\lambda_{2 j+1}, \lambda_{2 j}\right], j=0, \ldots, n-1$.

(iii) $L y=z y$ has two linearly independent Floquet solutions for all $z \in \mathbb{C}$ with the exception of the $2 n+1$ values $\lambda_{0}, \ldots, \lambda_{2 n}$.

(iv) $q$ has $n$ movable Dirichlet eigenvalues, precisely one in each of the closures of the spectral gaps $\left(\lambda_{2 j}, \lambda_{2 j-1}\right), j=1, \ldots, n$.

As mentioned previously, the classical examples for finite-band potentials are the Lamé potentials $q(x)=-s(s+1) \wp\left(x+c ; \omega_{1}, \omega_{3}\right), n \in \mathbb{N}$, with $c=\omega_{3}$ purely imaginary and $\omega_{1}$ real. However, Lamé potentials are algebro-geometric for general choices of the half-periods as well as for general choices of $c \in \mathbb{C}$. This suggests the study of complex-valued potentials with inverse square singularities as in [97] and [232], and we will subsequently report on some of these results.

Let $q$ be a complex-valued, periodic function with period $\Omega>0$, which is locally integrable on $\mathbb{R} \backslash \Sigma$, where $\Sigma \in \mathbb{R}$ is a discrete set (i.e., a set without finite accumulation points). Moreover, $q$ is assumed to be meromorphic near each $\xi \in \Sigma$ with principal part $-s(s+1) /(x-\xi)^{2}$, where $s=s(\xi) \in \mathbb{N}$. Then one can define unique solutions of initial value problems of the differential equation $y^{\prime \prime}(x)+q(x) y(x)=z y(x)$ on $\mathbb{R} \backslash \Sigma$ (with initial conditions at $x_{0} \in \mathbb{R} \backslash \Sigma$ ) by analytic continuation around the singularities. Even though the potential is no longer continuous, Floquet theory (see Section 2.6) remains essentially unchanged. 
To apply Floquet theory we first introduce a basis in $\mathcal{Y}(z)$, the space of solutions of $y^{\prime \prime}(x)+q(x) y(x)=z y(x)$. Let $c\left(z, \cdot, x_{0}\right), s\left(z, \cdot, x_{0}\right) \in \mathcal{Y}(z)$ be defined by the initial conditions $c\left(z, x_{0}, x_{0}\right)=s^{\prime}\left(z, x_{0}, x_{0}\right)=1$ and $c^{\prime}\left(z, x_{0}, x_{0}\right)=s\left(z, x_{0}, x_{0}\right)=0$ (prime denoting the derivative with respect to the second variable). Let $\rho_{ \pm}$denote the Floquet multipliers of $y^{\prime \prime}(x)+q(x) y(x)=z y(x)$, that is, the eigenvalues of the translation operator $\mathcal{T}_{\Omega}(z)$. An important role is played by $\operatorname{tr}\left(\mathcal{T}_{\Omega}(z)\right)$, which is sometimes called the Floquet discriminant and which, in our basis, is given by

$$
\operatorname{tr}\left(\mathcal{T}_{\Omega}(z)\right)=c\left(z, x_{0}+\Omega, x_{0}\right)+s^{\prime}\left(z, x_{0}+\Omega, x_{0}\right) .
$$

Since the trace is of course independent of the chosen basis in $\mathcal{Y}(z)$, the dependence of the right-hand side on $x_{0}$ is only apparent. The Floquet solutions may be expressed as

$$
f_{ \pm}\left(z, x_{0}, x\right)=s\left(z, x_{0}+\Omega, x_{0}\right) c\left(z, x_{0}, x\right)+\left(\rho_{ \pm}-c\left(z, x_{0}+\Omega, x_{0}\right)\right) s\left(z, x_{0}, x\right),
$$

and their Wronskian is given by

$$
W\left(f_{+}\left(z, \cdot, x_{0}\right), f_{-}\left(z, \cdot, x_{0}\right)\right)=-s\left(z, x_{0}+\Omega, x_{0}\right) \sqrt{\left(\operatorname{tr}\left(\mathcal{T}_{\Omega}(z)\right)\right)^{2}-4} .
$$

Next, consider the function

$$
g(z, x)=\frac{f_{+}\left(z, x, x_{0}\right) f_{-}\left(z, x, x_{0}\right)}{W\left(f_{+}\left(z, \cdot, x_{0}\right), f_{-}\left(z, \cdot, x_{0}\right)\right)} .
$$

As our notation for $g(z, x)$ suggests, the dependence of this function on $x_{0}$ is only apparent since $f_{ \pm}\left(z, \cdot, x_{1}\right)$ are just multiples of $f_{ \pm}\left(z, \cdot, x_{0}\right)$ and the right-hand side of (3.1.1) is independent of normalization. In particular, we may replace $x_{0}$ by $x$ in (3.1.1) to obtain

$$
g(z, x)=\frac{-s(z, x+\Omega, x)}{\sqrt{\left(\operatorname{tr}\left(\mathcal{T}_{\Omega}(z)\right)\right)^{2}-4}} .
$$

The function $s(\cdot, x+\Omega, x)$ is an entire function of order of growth $1 / 2$. The zeros of $s(\cdot, x+\Omega, x)$ are the Dirichlet eigenvalues of $d^{2} / d x^{2}+q$ on the interval $[x, x+\Omega]$, and their order, which we denote by $d(z, x)$, is the algebraic multiplicity of the corresponding Dirichlet eigenvalue (we set $d(z, x)=0$ if $s(z, x+\Omega, x) \neq 0$ ). We also introduce $d_{i}(z)=\min \{d(z, x) \mid x \in \mathbb{R} \backslash \Sigma\}$ and $d_{m}(z, x)=d(z, x)-d_{i}(z)$, the immovable and movable parts of $d(z, x)$, respectively. The quantity $\sum_{z \in \mathbb{C}} d_{m}(z, x)$, which is independent of $x$, is called the number of movable Dirichlet eigenvalues. Using Hadamard's factorization theorem we write $g(z, x)=F(z, x) D(z)$, collecting in $F$ the factors depending on $x$ and in $D$ the factors independent of $x$. Then the multiplicity of a zero $z$ of $D$ is just $d_{i}(z)$, while the multiplicity of a zero $z$ of $F(\cdot, x)$ is $d_{m}(z, x)$. One then obtains from Appell's equation (2.9.3) that

$$
F^{\prime}(z, x)^{2}-2 F(z, x) F^{\prime \prime}(z, x)-4(q-z) F(z, x)^{2}=\left(\left(\operatorname{tr}\left(\mathcal{I}_{\Omega}(z)\right)\right)^{2}-4\right) / D(z)^{2} .
$$

Recall that a zero $z$ of $\left(\operatorname{tr}\left(\mathcal{T}_{\Omega}(z)\right)\right)^{2}-4$ is an (anti-)periodic eigenvalue whose multiplicity we denote by $p(z)$. Since the left-hand side of equation (3.1.2) is entire as a function of $z$, we obtain the following result.

Theorem 3.2. There exists an entire function $\underline{R}$ such that $\left(\operatorname{tr}\left(\mathcal{T}_{\Omega}(z)\right)\right)^{2}-4=$ $\underline{R}(z)[0] D(z)^{2}$. In particular, $p(z)-2 d_{i}(z) \geq 0$ for every $z \in \mathbb{C}$. 
There are, at most, countably many points where $p(z)>0$ since these points are isolated. Therefore, there are at most countably many points where $p(z)-2 d_{i}(z)>$ 0 . These include all algebraically simple (anti-)periodic eigenvalues (where $p(z)=1$ ) but may well include other points too.

We call a Floquet solution $\psi\left(z_{0}, \cdot\right)$ of $y^{\prime \prime}(x)+q(x) y(x)=z_{0} y(x)$ regular if there exist Floquet solutions $\psi(z, \cdot)$ of $y^{\prime \prime}(x)+q(x) y(x)=z y(x)$, which converge pointwise to $\psi\left(z_{0}, \cdot\right)$ as $z$ tends to $z_{0}$. It was shown in [232] that the set of regular Floquet solutions forms a line bundle on the topological space $M_{F}$ obtained from the curve $\rho^{2}-\left(\operatorname{tr}\left(\mathcal{T}_{\Omega}(z)\right)\right) \rho+1=0$ by desingularization at all points where $p(z)=2 d_{i}(z)>0$. The space $M_{F}$ can be viewed as a double cover of the complex plane branched precisely at all points $z$ where $p(z)-2 d_{i}(z)>0$. In particular, the equation $y^{\prime \prime}(x)+$ $q(x) y(x)=z y(x)$ has two linearly independent regular Floquet solutions if and only if $p(z)-2 d_{i}(z)=0$. In other words, $p(z)-2 d_{i}(z)>0$ indicates a defect in the structure of regular Floquet solutions. The number $\operatorname{def}(q)=\sum_{z \in \mathbb{C}}\left(p(z)-2 d_{i}(z)\right)=$ $\operatorname{deg}(\underline{R})$, which is a positive integer or infinity, will therefore subsequently be called the Floquet defect of $q$. When $q$ is real-valued and nonsingular, then $p(z)-2 d_{i}(z)$ is always zero, except when $p(z)=1$, which forces $d_{i}(z)=0$. In this case $\operatorname{def}(q)$ counts the number of points $z \in \mathbb{C}$ where only one linearly independent Floquet solution exists. In general, however, $y^{\prime \prime}(x)+q(x) y(x)=z y(x)$ may have two linearly independent Floquet solutions with only one being regular.

Theorem 3.3. The following statements are equivalent:

(1) $\operatorname{def}(q)=2 n+1$.

(2) There are $n$ movable Dirichlet eigenvalues.

(3) There exists a differential expression $P_{2 n+1}$ of order $2 n+1$ but none of smaller odd order commuting with $L$. This differential expression satisfies $P_{2 n+1}^{2}=$ $R_{2 n+1}(z)=\prod_{z \in \mathbb{C}}(L-z)^{p(z)-2 d_{i}(z)}$ and hence $q$ is algebro-geometric.

Sketch of proof. Generally $p(z) \leq 2$ and $d\left(z, x_{0}\right) \leq 1$ for all suitably large $z$. Moreover, asymptotically, any Dirichlet eigenvalue is close to two (anti-)periodic eigenvalues. Therefore, $\operatorname{def}(q)=2 n+1$ implies $d_{m}\left(z, x_{0}\right)$ is different from zero for only finitely many $z \in \mathbb{C}$ and $\sum_{z \in \mathbb{C}} d_{m}(z, x)$, which equals the degree of $F\left(\cdot, x_{0}\right)$, must be finite. Equation (3.1.2) then yields $\operatorname{deg} F\left(\cdot, x_{0}\right)=n$. Hence (1) implies (2). The converse of this follows immediately from equation (3.1.2).

Differentiation of equation $(3.1 .2)$ shows that the third criterion after Definition 3.1 is satisfied when $F\left(\cdot, x_{0}\right)$ is a polynomial. Hence (2) implies (3). We remark here that $R_{2 n+1}$ is a constant multiple of $\underline{R}$. To prove that (3) implies (2) one has to show that the zeros of the function $F_{n}$ defined by $P_{2 n+1}$ are precisely the movable Dirichlet eigenvalues of $L$. This follows from applying $P_{2 n+1}$ given by (2.3.2) successively to the generalized Dirichlet eigenfunctions (i.e., the eigenfunctions corresponding to the algebraic eigenspace) associated with the movable Dirichlet eigenvalues of $L$ (cf. [232] for more details).

Remark 3.4. The quantity $p(z)-2 d_{i}(z)$ proves to be of utmost importance. Determining the points where it takes on positive values, one finds that this quantity then governs the structure of the line bundle of regular Floquet solutions and determines the entire function $\underline{R}(z)=\left(\left(\operatorname{tr}\left(\mathcal{T}_{\Omega}(z)\right)\right)^{2}-4\right) / D(z)^{2}$, which defines the algebraic curve associated with $q$ if $q$ is algebro-geometric and $\underline{R}$ is a polynomial. When $q$ is real-valued and locally integrable on $\mathbb{R}$, then geometric and algebraic multiplicities of Dirichlet and (anti-)periodic boundary value problems coincide. 
Therefore, $0 \leq p(z) \leq 2$ and $0 \leq d\left(z, x_{0}\right) \leq 1$. Thus, $d_{i}(z)=1$ enforces $p(z)=2$. In addition, if $p(z)=2$, then all solutions are (anti-)periodic, implying $d_{i}(z)=1$. In this case the questions "When is $p(z)-2 d_{i}(z)>0$ ?" and "When is $p(z)=1$ ?" are equivalent. Hence, in determining the edges of the spectral gaps, the role played by the Dirichlet eigenvalues and, in particular, the distinction between movable and immovable Dirichlet eigenvalues are secondary in the case of real-valued locally integrable potentials.

Remark 3.5. One may also show that every algebro-geometric potential is a finiteband potential; that is, the conditional stability set (which coincides with the spectrum of $H$ when no singularities are present) consists of finitely many regular analytic arcs.

When $q$ is real and has no singularities, the converse is also true (Dubrovin [52]). However, in general this is not the case as the following example shows. Let $q(x)=$ $\mathrm{e}^{2 i x}$. Then a fundamental system of solutions of $y^{\prime \prime}(x)+q(x) y(x)=-\nu^{2} y(x)$ is given in terms of Bessel functions (cf. [3], Ch. 9) by $y_{1}(x)=J_{\nu}\left(i \mathrm{e}^{i x}\right), y_{2}(x)=Y_{\nu}\left(i \mathrm{e}^{i x}\right)$. Note that $y_{1}$ is always a Floquet solution with multiplier $\mathrm{e}^{\nu \pi i}$. Hence $z=-\nu^{2}$ is in the conditional stability set if and only if $\nu \in \mathbb{R}$. Consequently, $\mathcal{S}(L)=(-\infty, 0]$. However, $s\left(\nu^{2}, x_{0}+\pi, x_{0}\right)=\pi J_{\nu}\left(i \mathrm{e}^{i x_{0}}\right) J_{-\nu}\left(i \mathrm{e}^{i x_{0}}\right)$, which is entire as a function of $x_{0}$. Hence $d_{i}\left(-\nu^{2}\right)=0$ for all $\nu \in \mathbb{C}$; that is, every Dirichlet eigenvalue is movable. Thus, $\operatorname{def}(q)=\infty$, and hence $q$ is not algebro-geometric. More general examples of this type have been studied systematically by Gasymov [79], [80], Guillemin and Uribe [105], and Pastur and Tkachenko [173], [174].

\subsection{Picard-KdV Potentials.}

Definition 3.6. Let $q$ be an elliptic function. Then $q$ is called a Picard-KdV potential (or simply a Picard potential) if the equation $y^{\prime \prime}(x)+q(x) y(x)=z y(x)$ has a meromorphic fundamental system of solutions with respect to $x$ for all values of the spectral parameter $z \in \mathbb{C}$.

Theorem 3.7. If $q$ is a Picard potential, then it may be represented as

$$
q(x)=C-\sum_{j=1}^{m} s_{j}\left(s_{j}+1\right) \wp\left(x-b_{j}\right)
$$

for suitable integers $s_{1}, \ldots, s_{m}$ and complex numbers $C, b_{1}, \ldots, b_{m}$, where the $b_{j}$ are pairwise distinct $\bmod \Delta$.

Sketch of proof. Every singularity of $y^{\prime \prime}(x)+q(x) y(x)=z y(x)$ must be a regular singular point with integer indices. From the partial fraction expansion for elliptic functions (Theorem 2.2) one obtains

$$
q(x)=C-\sum_{j=1}^{m}\left(s_{j}\left(s_{j}+1\right) \wp\left(x-b_{j}\right)+B_{j} \zeta\left(x-b_{j}\right)\right) .
$$

In a vicinity of $b_{j}$ there is a solution of the form

$$
\psi(x)=\left(x-b_{j}\right)^{-s_{j}} \sum_{k=0}^{\infty} \beta_{k}\left(x-b_{j}\right)^{k},
$$

where $\beta_{0}=1$. Next we use the Frobenius method to show that $B_{j}=0$. Let

$$
q(x)=\frac{-s_{j}\left(s_{j}+1\right)}{\left(x-b_{j}\right)^{2}}+\frac{B_{j}}{x-b_{j}}+\sum_{k=0}^{\infty} C_{j, k}\left(x-b_{j}\right)^{k}
$$


and insert $\psi$ into the differential equation $y^{\prime \prime}+(q-z) y=0$ to get

$$
\begin{aligned}
0= & f\left(-s_{j}\right)\left(x-b_{j}\right)^{-s_{j}}+\left\{f\left(1-s_{j}\right) \beta_{1}+G_{1}\right\}\left(x-b_{j}\right)^{1-s_{j}} \\
& +\cdots+\left\{f\left(k-s_{j}\right) \beta_{k}+G_{k}\right\}\left(z-b_{j}\right)^{k-s_{j}}+\cdots,
\end{aligned}
$$

where $f(\ell)=\left(\ell+s_{j}\right)\left(\ell-s_{j}-1\right)$ and

$$
G_{k}=B_{j} \beta_{k-1}+\left(C_{j, 0}-z\right) \beta_{k-2}+C_{j, 1} \beta_{k-3}+\cdots+C_{j, k-2} \beta_{0} .
$$

Now $f\left(-s_{j}\right)=0$ and $\beta_{1}, \ldots, \beta_{2 s_{j}}$ may be determined recursively so that the coefficients of $\left(x-b_{j}\right)^{1-s_{j}}, \ldots,\left(x-b_{j}\right)^{s_{j}}$ vanish. But since $f\left(s_{j}+1\right)=0$, the coefficient of $\left(x-b_{j}\right)^{s_{j}+1}$ is just $G_{2 s_{j}+1}$, which therefore must vanish for all $z \in \mathbb{C}$. On the other hand, if $B_{j} \neq 0$, one can show by induction that $G_{2 s_{j}+1}$ is a polynomial in $z$ of degree $s_{j}$. This contradiction completes the proof.

If $q$ is a Picard potential, then, by Picard's theorem (Theorem 2.7), the equation $y^{\prime \prime}(x)+q(x) y(x)=z y(x)$ has at least one solution which is elliptic of the second kind. Using Theorem 2.4 and the special structure of the Picard potential (3.2.1), one may represent this solution as

$$
\psi_{a(z)}(x)=\frac{\prod_{j=1}^{s} \sigma\left(x-a_{j}(z)\right)}{\prod_{j=1}^{m} \sigma\left(x-b_{j}\right)^{s_{j}}} \exp \left(\lambda_{a(z)} x\right)
$$

where $s=\sum_{j=1}^{m} s_{j}$ and $a(z)=\left(a_{1}(z), \ldots, a_{s}(z)\right)$. At $b_{j}$ the function $\psi_{a}$ has a pole of order $s_{j}$ or a zero of order $s_{j}+1$. For later notational purposes we allow for $s_{j}=0$, in which case $\psi_{a}$ has either no pole and no zero or a simple zero. For subsequent use we define

$$
\begin{aligned}
& M_{1}=\left\{j \in\{1, \ldots, m\} \mid \operatorname{ord}_{b_{j}}\left(\psi_{a}\right)=-s_{j}\right\}, \\
& M_{2}=\left\{j \in\{1, \ldots, m\} \mid \operatorname{ord}_{b_{j}}\left(\psi_{a}\right)=s_{j}+1\right\} .
\end{aligned}
$$

The function $\psi_{a}$ is a solution of $y^{\prime \prime}(x)+q(x) y(x)=z y(x)$ if and only if

$$
\begin{aligned}
& \lambda_{a}+\sum_{j=1}^{s} \zeta\left(b_{r}-a_{j}\right)-\sum_{\substack{j=1 \\
j \neq r}}^{m} s_{j} \zeta\left(b_{r}-b_{j}\right)=0, \\
& z=C-\sum_{j=1}^{s}\left(1-2 s_{r}\right) \wp\left(b_{r}-a_{j}\right)-\sum_{\substack{j=1 \\
j \neq r}}^{m} s_{j}\left(s_{j}+2 s_{r}\right) \wp\left(b_{r}-b_{j}\right),
\end{aligned}
$$

where $r$ is chosen such that $s_{r} \neq 0$ and $\operatorname{ord}_{b_{r}}\left(\psi_{a}\right)=-s_{r}$, which is always possible. In the case of a Lamé potential these conditions are recorded, for instance, by Burkhardt [32]. The subscript $a$ in $\lambda_{a}$ expresses the dependence of $\lambda_{a}$ on $a=$ $\left(a_{1}, \ldots, a_{s}\right)$, which in turn depends on $z$.

In [95] we have developed a method for even Picard potentials (i.e., potentials $q$ satisfying $q\left(x_{0}+x\right)=q\left(x_{0}-x\right)$ for some $\left.x_{0} \in \mathbb{C}\right)$ to determine all points where two regular Floquet solutions fail to exist. (For simplicity we will assume $x_{0}=0$ from now on.) First note that an even Picard potential is of the form

$$
q(x)=C-\sum_{k=1}^{4} s_{k}\left(s_{k}+1\right) \wp\left(x-\omega_{k}\right)-\sum_{k=1}^{\tilde{m}} r_{k}\left(r_{k}+1\right)\left[\wp\left(x-b_{k}\right)+\wp\left(x+b_{k}\right)\right],
$$


where the $b_{k}$ are pairwise distinct and different from half-periods, the $s_{k}$ are nonnegative and the $r_{k}$ are positive. If $\tilde{m}=0$, that is, if

$$
q(x)=C-\sum_{k=1}^{4} s_{k}\left(s_{k}+1\right) \wp\left(x-\omega_{k}\right),
$$

then $q$ is called a Treibich-Verdier potential following the work of Verdier [229] and Treibich and Verdier [217], [218], [219], [220], [221], [222], [223], [224], [225], [226]. If, in addition, only one of the numbers $s_{k}$ is different from zero, then $q$ is a Lamé potential. In order to make use of previous results we will adopt the following notation: $m=2 \tilde{m}+4, b_{k+\tilde{m}}=-b_{k}, s_{k+\tilde{m}}=r_{k}$ for $k=1, \ldots, \tilde{m}$, and $b_{k+2 \tilde{m}}=\omega_{k}$, $s_{k+2 \tilde{m}}=s_{k}$ for $k=1, \ldots, 4$. Let $\hat{s}$ be the number of $a_{j}$ 's which do not appear in $\left\{b_{1}, \ldots, b_{m}\right\}$.

If $q$ is an even Picard potential of the type (3.2.5), and if $\psi_{a}$ given by (3.2.2) is a solution of the differential equation $y^{\prime \prime}(x)+q(x) y(x)=z y(x)$, then so is the function $\psi_{-a}$, which is obtained by replacing every $a_{j}$ with $-a_{j}$ in (3.2.2) and (3.2.3), since $\psi_{-a}(x)=(-1)^{s_{1}+s_{2}+s_{3}} \psi_{a}(-x)$.

Next we compute the Wronskian of the two solutions $\psi_{a}$ and $\psi_{-a}$. One obtains an expression which involves $x$, but since the Wronskian does not depend on $x$, one may evaluate it anywhere. Choosing $x=a_{\ell}$ for any $a_{\ell}$ which does not appear in $\left\{b_{1}, \ldots, b_{m}\right\}$, one finds that the Wronskian is a nonzero multiple of

$$
\frac{\sigma\left(2 a_{\ell}\right)}{\sigma\left(a_{\ell}-b_{r}\right) \sigma\left(a_{\ell}+b_{r}\right)} \prod_{\substack{j=1 \\ j \neq \ell}}^{\hat{s}} \sigma\left(a_{\ell}-a_{j}\right) \sigma\left(a_{\ell}+a_{j}\right) .
$$

Since $a_{\ell}$ is different from all the $b_{j}$ and, in particular, different from the half-periods, one infers $\sigma\left(2 a_{\ell}\right) \neq 0$. Also $a_{\ell} \neq a_{k}$ if $k \neq \ell$. Therefore we find that the Wronskian is zero if and only if $\sigma\left(a_{\ell}+a_{j}\right)=0$ for some $j \in\{1, \ldots, \ell-1, \ell+1, \ldots, \hat{s}\}$ and hence $a_{j}=-a_{\ell}(\bmod \Delta)$. In particular, we find that the number $\hat{s}$ is even and define $d=\hat{s} / 2$.

Choosing now $x=b_{\ell}$ for any $\ell \in M_{2}$, one can write the Wronskian as a nonzero multiple of

$$
\frac{\sigma\left(2 b_{\ell}\right)^{2 s_{\ell}+1}}{\sigma\left(b_{\ell}-b_{r}\right) \sigma\left(b_{\ell}+b_{r}\right)} \frac{\prod_{\substack{j \in M_{2} \\ j \neq \ell}} \sigma\left(b_{\ell}-b_{j}\right)^{2 s_{j}+1} \sigma\left(b_{\ell}+b_{j}\right)^{2 s_{j}+1}}{\prod_{\substack{j=1 \\ j \neq \ell}}^{m} \sigma\left(b_{\ell}-b_{j}\right)^{2 s_{j}}},
$$

which is zero if and only if $b_{\ell}$ is a half-period or if there is a $j \in M_{2}$ such that $b_{j}=-b_{\ell}(\bmod \Delta)$.

In summary we have found the following: if $\psi_{a}$ and $\psi_{-a}$ are linearly dependent solutions of $\psi^{\prime \prime}+q \psi=z \psi$, then some of the numbers $a_{1}, \ldots, a_{s}$ may be half-periods while all others appear in pairs $\left(a_{j}, a_{\ell_{j}}\right)$ with $a_{\ell_{j}}=-a_{j}$. Moreover, if $a_{j}$ is equal to a half-period $\omega_{k}$, which is a pole of $q$ of the form $-r_{k}\left(r_{k}+1\right) /\left(z-\omega_{k}\right)^{2}$, then there are exactly $2 r_{k}+1$ of the $a_{\ell}$ which are equal to this half-period. If $a_{j}$ equals a pole $b_{\ell}$ of the form $-s_{\ell}\left(s_{\ell}+1\right) /\left(z-b_{\ell}\right)^{2}$, where $b_{\ell}$ is not a half-period, then there are exactly $2 s_{\ell}+1$ of the $a_{m}$ which are equal to this pole and exactly $2 s_{\ell}+1$ other $a_{m}$ 's which are equal to the pole $-b_{\ell}$.

This information is now being used to rewrite the solution $\psi_{a}$ of $\psi^{\prime \prime}+q \psi=z \psi$ for those values of the spectral parameter $z$ where $W\left(\psi_{a}, \psi_{-a}\right)=0$ as a product of two functions. The first function is fixed, depending only on the poles of the potential $q$, on the half-periods, and on the exponents associated with these. The second 
function is a polynomial in $\wp(x)$, whose coefficients depend on those $a_{j}$ which are neither half-periods nor poles of $q$ and which are as yet undetermined. According to the above argument there must be an even number, $2 d$, of those, and half of them are just negatives of the other half.

Hence we define $t_{\ell}=\operatorname{ord}_{b_{\ell}}\left(\psi_{a}\right)$ for $\ell=1, \ldots, 2 \tilde{m}+4$ and obtain $\psi_{a}(x)=$ $f(x) Q(\wp(x))$, where

$$
\begin{aligned}
f(x) & =\mathrm{e}^{\lambda_{a} x}\left(\prod_{k=1}^{4} \sigma\left(x-\omega_{k}\right)^{t_{k+2 \tilde{m}}}\right)\left(\prod_{\ell=1}^{2 \tilde{m}} \sigma\left(x-b_{\ell}\right)^{t_{\ell}}\right) \sigma(x)^{2 d}, \\
Q(\wp(x)) & =\prod_{j=1}^{d}\left(\wp(x)-\wp\left(a_{j}\right)\right)=\sum_{j=0}^{d} c_{j} \wp(x)^{j} .
\end{aligned}
$$

Here we used the fact that $\sigma\left(z-a_{j}\right) \sigma\left(z+a_{j}\right)=-\sigma(z)^{2} \sigma\left(a_{j}\right)^{2}\left(\wp(z)-\wp\left(a_{j}\right)\right)$. Moreover, we dropped the non-zero constant factor $(-1)^{d} \prod_{j=1}^{d} \sigma\left(a_{j}\right)^{2}$. This yields

$$
\begin{aligned}
& \psi_{a}^{\prime \prime}(x)+q(x) \psi_{a}(x) \\
= & f(x)\left\{\sum_{k=0}^{d} \sum_{j=0}^{d} S_{k+1, j+1} c_{j} \wp(x)^{k}+\frac{\sum_{k=1}^{\tilde{m}} \sum_{j=0}^{d} T_{k, j+1} c_{j} \wp(x)^{k-1}}{\prod_{j=1}^{\tilde{m}}\left(\wp(z)-\wp\left(b_{j}\right)\right)}\right\}
\end{aligned}
$$

for suitable constants $S_{j, k}$ and $T_{j, k}$ depending on $q$ and the numbers $t_{\ell}$. Let $S$ and $T$ be the matrices with entries $S_{j, k}$ and $T_{j, k}$.

Hence we obtain a solution $\psi_{a}$ of the equation $y^{\prime \prime}(x)+q(x) y(x)=z y(x)$ satisfying $W\left(\psi_{a}, \psi_{-a}\right)=0$ if and only if

$$
S \gamma=z \gamma \text { and } T \gamma=0
$$

where $\gamma=\left(c_{0}, \ldots, c_{d}\right)^{T}$.

For any given even Picard potential there are several (but finitely many) choices to distribute some (or all) of the parameters $a_{1}, \ldots, a_{s}$ among the half-periods and/or poles of $q$. Accordingly, there are several (but finitely many) of the above described constraint eigenvalue problems to solve in order to find all the values of the spectral parameter $z \in \mathbb{C}$ where $W\left(\psi_{a}, \psi_{-a}\right)=0$. In each case there are only finitely many eigenvalues of the associated matrix $S$, some (or possibly all) of which may be in contradiction to the constraint $T \gamma=0$. Thus, we proved the following result.

Theorem 3.8. Let $q$ be an even Picard potential. Then, for every complex number $z$, the differential equation $y^{\prime \prime}(x)+q(x) y(x)=z y(x)$ has a solution $\psi_{a}$ of the form (3.2.2); that is, a solution which is elliptic of the second kind. Similarly, the function $\psi_{-a}$ is a solution of the same equation (for the same value of $z$ ) and also elliptic of the second kind. With respect to any period of $q$, the functions $\psi_{a}$ and $\psi_{-a}$ are regular Floquet solutions. For all but a finite number of values of $z \in \mathbb{C}$ these two solutions are linearly independent and therefore $p(z)-2 d_{i}(z)=0$. Hence $\operatorname{def}(q)<$ $\infty$; that is, $q$ is algebro-geometric.

In the case of a Treibich-Verdier potential

$$
q(x)=-\sum_{j=1}^{4} s_{j}\left(s_{j}+1\right) \wp\left(x-\omega_{j}\right), \quad s_{j} \in \mathbb{N} \cup\{0\}, j=1, \ldots, 4,
$$

the matrix $T$ is absent, and therefore one has to find only the eigenvalues of $S$ for all possible choices of the numbers $t_{\ell}$. This was performed in [93] for Lamé 
potentials and in [94] for Treibich-Verdier potentials. The arithmetic genus of the curve $P^{2}=R(L)$ associated with $q$ is given in the following table, where $s=s_{1}+s_{2}+s_{3}+s_{4}$ (and, without loss of generality, $s_{1} \geq s_{2} \geq s_{3} \geq s_{4} \geq 0$ ).

\begin{tabular}{|c|c|c|c|}
\hline$s$ & & \# of finite branch points & genus \\
\hline even & $s_{2}+s_{3} \leq s_{1}+s_{4}$ & $2 s_{1}+1$ & $s_{1}$ \\
\hline even & $s_{2}+s_{3} \geq s_{1}+s_{4}$ & $s_{1}+s_{2}+s_{3}-s_{4}+1$ & $\frac{s}{2}-s_{4}$ \\
\hline odd & $s_{2}+s_{3}+s_{4}<s_{1}$ & $2 s_{1}+1$ & $s_{1}$ \\
\hline odd & $s_{2}+s_{3}+s_{4}>s_{1}$ & $s_{1}+s_{2}+s_{3}+s_{4}+2$ & $\frac{s+1}{2}$ \\
\hline
\end{tabular}

Shortly after this table appeared in our paper [94], Armando Treibich kindly sent us the following formula for the arithmetic genus of the Treibich-Verdier curve. It is given by

$$
\max \left\{2 s_{1}, s+1-\left(1+(-1)^{s}\right)\left(s_{4}+(1 / 2)\right)\right\} / 2
$$

in agreement with our column on the right.

Remark 3.9. We briefly return to the Picard potential $q(x)$ in (3.2.1). Assuming first the special case where $s_{j}=1, j=1, \ldots, m$, that is,

$$
q(x)=-2 \sum_{j=1}^{m} \wp\left(x-b_{j}\right), \quad b_{k} \neq b_{\ell}(\bmod \Delta) \text { for } k \neq \ell,
$$

Theorem 2.5 yields Krichever's ansatz (2.8.3) in [135], that is,

$$
\psi_{a(z)}(x)=\exp \left(\lambda_{a(z)} x\right) \prod_{j=1}^{m} \frac{\sigma\left(x-a_{j}(z)\right)}{\sigma\left(x-b_{j}\right)}=e^{\kappa(z) x} \sum_{j=1}^{m} A_{j}(z) \Phi\left(x-b_{j}, \rho(z)\right),
$$

where

$$
\Phi(x, \rho)=e^{\zeta(\rho) x} \sigma(x-\rho) \sigma(x)^{-1} \sigma(-\rho)^{-1}
$$

and where $\kappa, A_{j}$ and $\rho_{j}$ are suitably chosen.

In the general case, where

$$
q(x)=-\sum_{j=1}^{m} s_{j}\left(s_{j}+1\right) \wp\left(x-b_{j}\right), \quad b_{k} \neq b_{\ell}(\bmod \Delta) \text { for } k \neq \ell,
$$

one can use an extension of Theorem 2.5 (using, e.g., l'Hospital's rule as several $b_{j}$ 's are confluenting into a $b_{j_{0}}$ and converting $b$-derivatives into an $x$-derivative) to arrive at the corresponding analog

$$
\begin{aligned}
\psi_{a(z)}(x) & =\exp \left(\lambda_{a(z)} x\right) \frac{\prod_{j=1}^{s} \sigma\left(x-a_{j}(z)\right)}{\prod_{j=1}^{m} \sigma\left(x-b_{j}\right)^{s_{j}}} \\
& =e^{\kappa(z) x} \sum_{j=1}^{m} \sum_{k=0}^{s_{j}-1} A_{j, k}(z) \Phi^{(k)}\left(x-b_{j}, \rho(z)\right), \quad s=\sum_{j=1}^{m} s_{j}
\end{aligned}
$$

of (3.2.7). The extended ansatz (3.2.9) was recently used by Enol'skii and Eilbeck [58], [63], Enol'skii and Kostov [64], [129], in the context of Treibich-Verdier potentials. The special case $m=1, s_{1}=2$ can be found in Hermite [112], pp. 374-377 (see also Forsyth [75], pp. 475-476); the general case (3.2.9) is discussed in Krause's monograph [131], Vol. 1, pp. 292-296; Vol. 2, pp. 183, 259-264. 
We emphasize that Picard's theorem, Theorem 2.7, yields the $\sigma$-function representations of $\psi_{a(z)}(x)$ in (3.2.7) and (3.2.9) and hence Krichever's ansatz (2.8.3) and its extension (3.2.9). In particular, an alternative characterization of all stationary elliptic KdV and AKNS potentials to the one provided in Theorem 3.12 and Theorem 4.8, respectively, can be based on these observations (cf. [100]). In the remainder of this review we do not pursue this avenue but stress Floquet-theoretic and Green's function methods instead.

\subsection{Necessary Conditions for a KdV Potential to Be Algebro-Geometric.}

Theorem 3.10. Suppose $q$ is an algebro-geometric KdV potential. Then $(L y)(x)=$ $y^{\prime \prime}(x)+q(x) y(x)=z y(x)$ has a meromorphic fundamental system of solutions with respect to $x$ for all values of the spectral parameter $z \in \mathbb{C}$.

Sketch of proof. First one shows that every pole of $q$ is of second order. This follows since only in this case is there a balance between the growth of the order of the poles of $f_{j}^{\prime \prime \prime}$ and $4 q f_{j}^{\prime}+2 q^{\prime} f_{j}$ considering the recursion relation (2.3.3). Such a balance is necessary since eventually all $f_{j}$ vanish. Next one shows by a similar argument that the leading term of the Laurent expansion of $q$ about a pole $x_{0}$ must be $-s(s+1) /\left(x-x_{0}\right)^{2}$ for some integer $s$. This guarantees that the exponents of the singularity $x_{0}$ of the differential equation $y^{\prime \prime}(x)+q(x) y(x)=z y(x)$ are integers. Finally one has to show the absence of logarithmic terms in the solutions of $y^{\prime \prime}(x)+q(x) y(x)=z y(x)$ which follows from a careful analysis using the Frobenius method. More details are provided in [233].

We note that $\tau$-function results in Segal and Wilson [193] imply Theorem 3.10. (In the case of nonsingular curves $\mathcal{K}_{n}$ this simply follows from the standard thetafunction representation of the Baker-Akhiezer function.) While Segal and Wilson rely on loop group techniques (and study the Gelfand-Dickey hierarchy), the above proof represents a completely elementary alternative for the KdV hierarchy.

\subsection{Sufficient Conditions for a KdV Potential to Be Algebro-Geometric - Characterizations of Elliptic, Simply Periodic, and Rational Stationary} KdV Solutions.

Theorem 3.11. $q$ is an algebro-geometric potential if, for each $z \in \mathbb{C}, y^{\prime \prime}(x)+$ $q(x) y(x)=z y(x)$ has a meromorphic fundamental system of solutions with respect to $x$ and if one of the following three conditions is satisfied.

(i) $q$ is rational and bounded near infinity.

(ii) $q$ is simply periodic with period $\Omega$ and there exists a positive number $R$ such that $q$ is bounded in $\{x \in \mathbb{C}|| \operatorname{Im}(x / \Omega) \mid \geq R\}$.

(iii) $q$ is elliptic.

Sketch of proof. We will first sketch the proof in [97] which treats the case where $q$ is elliptic and prominently uses the double periodicity of $q$.

Let $q$ be an elliptic function with fundamental periods $2 \omega_{1}$ and $2 \omega_{3}$. Assume, without loss of generality, that $\operatorname{Im}\left(\omega_{3} / \omega_{1}\right)>0$. Introduce $t_{j}=\omega_{j} /\left|\omega_{j}\right|$ and define

$$
q_{j}(x)=t_{j}^{2} q\left(t_{j} x\right) .
$$

The transformation $\xi=t_{j} x, \psi(\xi)=w(x)$ transforms $\psi^{\prime \prime}+q \psi=z \psi$ into $w^{\prime \prime}+q_{j} w=$ $t_{j}^{2} z w$.

Note that $q_{j}$ has the period $2\left|\omega_{j}\right|$. With the aid of Rouché's theorem, one may determine the asymptotic distribution of the (anti-)periodic eigenvalues of $d^{2} / d x^{2}+q_{j}$ 
and verify that they all stay close to the real axis while their real parts tend to $-\infty$. (Never mind that $q_{j}$ may have inverse square singularities; see [232].) Equivalently, all $2 \omega_{j}$-(anti-)periodic eigenvalues of $d^{2} / d x^{2}+q$ lie in the half-strip $\Sigma_{j}$ given by

$$
\Sigma_{j}=\left\{z \in \mathbb{C}|| \operatorname{Im}\left(t_{j}^{2} z\right) \mid \leq C, \operatorname{Re}\left(t_{j}^{2} z\right) \leq C\right\}, \quad j=1,3
$$

for some constant $C>0$. Note that the angle between the strips $\Sigma_{1}$ and $\Sigma_{3}$ is positive and less than $2 \pi$ and therefore they intersect only in a finite part of the complex plane. Hence, for every point $z$ outside a sufficiently large compact disk, at least one of the translation operators $\mathcal{T}_{1}$ or $\mathcal{T}_{3}$ has distinct eigenvalues. Assuming, according to our hypothesis, that all solutions of $y^{\prime \prime}(x)+q(x) y(x)=z y(x)$ are meromorphic, we may invoke Picard's theorem to conclude that there are two linearly independent solutions which are elliptic of the second kind for any $z$ outside that disk. These solutions are Floquet solutions with respect to any period of $q$, and since the disk is compact, we have shown that there are at most finitely many points which lack two linearly independent Floquet solutions. Applying Theorem 3.3 then proves that $q$ is algebro-geometric.

Next we sketch the proof for the case when $q$ is rational. That proof can easily be extended for the simply periodic and elliptic potentials. In the latter case one uses the algebraic properties of elliptic functions rather than their double periodicity. For more details see [233].

Suppose that $q$ is rational and bounded at infinity. Let $z_{0}=\lim _{x \rightarrow \infty} q(x)$. From Halphen's theorem (Theorem 2.8) we obtain for $z \neq z_{0}$ the existence of linearly independent solutions

$$
y_{ \pm}(z, x)=R_{ \pm}(z, x) \exp \left( \pm \sqrt{z-z_{0}} x\right)
$$

where $R_{ \pm}(z, \cdot)$ are rational functions. The poles of $R_{ \pm}(z, \cdot)$ are determined as the singular points of the differential equation and their orders as the exponents of the corresponding singularities. Next define the function $g(z, x)=y_{+}(z, x) y_{-}(z, x)$. Then there exists a polynomial $v$ in $x$ such that

$$
v(x)^{2} g(z, x)=\sum_{j=0}^{d} c_{j}(z) x^{j} .
$$

Next note that the functions $v^{2} g(z, x), v^{3} g^{\prime}(z, x), v^{4} g^{\prime \prime}(z, x)$, and $v^{5} g^{\prime \prime \prime}(z, x)$ are polynomials in $x$ whose coefficients are homogeneous polynomials of degree one in $c_{0}, \ldots, c_{d}$. Also $v^{2} q$ and $v^{3} q^{\prime}$ are polynomials. Hence $v^{5}\left(g^{\prime \prime \prime}+4(q-z) g^{\prime}+2 q^{\prime} g\right)$ is also a polynomial in $x$, whose coefficients are homogeneous polynomials of degree one in $c_{0}, \ldots, c_{d}$. The coefficients of the polynomials $c_{\ell}$ in this last expression are polynomials in $z$ of degree at most one, that is,

$$
v^{5}\left(g^{\prime \prime \prime}+4(q-z) g^{\prime}+2 q^{\prime} g\right)=\sum_{j=0}^{N} \sum_{\ell=0}^{d}\left(\alpha_{j, \ell}+\beta_{j, \ell} z\right) c_{\ell} x^{j}
$$

for suitable numbers $N, \alpha_{j, \ell}$, and $\beta_{j, \ell}$ which only depend on $q$. From Appell's equation (2.9.2) it follows upon differentiation that the expression (3.4.1) vanishes identically. This gives rise to a homogeneous system of $N+1$ linear equations which has a nontrivial solution. Solving the system shows now that the coefficients $c_{\ell}$ are rational functions of $z$. Therefore

$$
g(z, x)=\frac{F(z, x)}{\gamma(z)}
$$


where $F(\cdot, x)$ and $\gamma$ are polynomials and $F(z, \cdot)$ is a rational function. Hence $q$ is algebro-geometric by item (3) following Definition 3.1.

Combining the results of this subsection and its preceding one yields the following explicit characterization of all elliptic algebro-geometric KdV potentials (a problem posed, for instance, by Novikov, Manakov, Pitaevskii, and Zakharov [169], p. 152), originally proven in [97] (see also [96], [99]).

Theorem 3.12. Let $q$ be an elliptic function. Then $q$ is an elliptic algebro-geometric KdV potential if and only if it is a Picard potential.

Similarly, these results characterize the stationary rational KdV potentials vanishing at infinity studied, for instance, by Adler and Moser [4], Airault, McKean, and Moser [5], Calogero [34], [35], Chudnovsky and Chudnovsky [38], [40], Grinevich [104], Krichever [134], Matveev [154], and Sokolov [206].

\section{Algebro-geometric and especially, elliptic AKNS potentials}

Most of the results in this section are taken from [98]. Since we take here a slightly different point of departure, the notation differs a bit from that in [98].

4.1. The AKNS Hierarchy. Let $L=J d / d x+Q(x)$, where

$$
J=\left(\begin{array}{cc}
i & 0 \\
0 & -i
\end{array}\right) \text { and } Q(x)=\left(\begin{array}{cc}
0 & -i q(x) \\
i p(x) & 0
\end{array}\right) .
$$

Note that $J^{2}=-I$, the $2 \times 2$ identity matrix, and that $J Q+Q J=0$. As mentioned in Section 2.3 one may consider the Lax pair $\left(P_{n+1}, L\right)$, where $P_{n+1}$ is a $2 \times 2$-matrixvalued differential expression of order $n+1$ such that $\left[P_{n+1}, L\right]$ is an operator of multiplication. Writing

$$
P_{n+1}=\sum_{\ell=0}^{n+1} C_{n+1-\ell}(x) L^{\ell},
$$

and utilizing the fact that the commutator $\left[C_{j}, L\right]$ may be written as $D_{j}(x) L+E_{j}(x)$, one finds that the condition that $\left[P_{n+1}, L\right]$ is an operator of multiplication yields $D_{j+1}(x)=-E_{j}(x)$ for $j=0, \ldots, n, D_{0}=0$, and $\left[P_{n+1}, L\right]=E_{n+1}(x)$. Note that $C_{j}(x)$ can be expressed as

$$
C_{j}(x)=k_{j}(x) I+v_{j}(x) J+W_{j}(x), \quad j=0, \ldots, n+1,
$$

where the $k_{j}(x)$ and $v_{j}(x)$ are scalar-valued and the matrices $W_{j}(x)$ have vanishing diagonal elements. Then $\left({ }^{\prime}=d / d x\right)$

$$
D_{j}=2 W_{j} \text { and } E_{j}=-W_{j} Q-Q W_{j}+v_{j}^{\prime} I-k_{j}^{\prime} J+2 v_{j} J Q-J W_{j}^{\prime} .
$$

Since $W_{j} Q+Q W_{j}$ is a multiple of the identity matrix $I, D_{j+1}+E_{j}=0$ shows that $k_{j}^{\prime}=0$ for all $j=0, \ldots, n+1$, and that the following recursion relation holds:

$$
\begin{aligned}
W_{0} & =0, \\
v_{j}^{\prime} I & =W_{j} Q+Q W_{j}, \quad W_{j+1}=\frac{1}{2} J\left(W_{j}^{\prime}-2 v_{j} Q\right), \quad j=0, \ldots, n+1 .
\end{aligned}
$$

One concludes

$$
\left[P_{n+1}, L\right]=2 v_{n+1} J Q-J W_{n+1}^{\prime} .
$$


Next, let

$$
\begin{aligned}
K_{n+1}(z) & =\sum_{j=0}^{n+1} k_{n+1-j} z^{j}, \\
V_{n+1}(z, x) & =\sum_{j=0}^{n+1} v_{n+1-j}(x) z^{j}, \\
\mathcal{W}_{n+1}(z, x) & =\sum_{j=1}^{n+1} W_{n+1-j}(x) z^{j},
\end{aligned}
$$

so that the recursion relation (4.1.2) becomes

$$
\begin{aligned}
& V_{n+1}^{\prime}(z, x) I=\mathcal{W}_{n+1}(z, x) Q(x)+Q(x) \mathcal{W}_{n+1}(z, x) \\
& \mathcal{W}_{n+1}^{\prime}(z, x)=2 V_{n+1}(z, x) Q(x)-2 z J \mathcal{W}_{n+1}(z, x)+J\left[P_{n+1}, L\right]
\end{aligned}
$$

Hence,

$$
\left[P_{n+1}, L\right]=2 z \mathcal{W}_{n+1}(z, x)+2 V_{n+1}(z, x) J Q(x)-J \mathcal{W}_{n+1}^{\prime}(z, x) .
$$

If $P_{n+1}$ is of order $n+1$, we define the $n^{\text {th }}$ order AKNS equations by

$$
\operatorname{AKNS}_{n}(Q)=Q_{t}-\left[P_{n+1}, L\right]=0 .
$$

The first few of these equations are

$$
\begin{aligned}
& Q_{t}=-v_{0} Q^{\prime}+2 c_{1} J Q, \\
& Q_{t}=-\frac{v_{0}}{2} J\left(Q^{\prime \prime}-2 Q^{3}\right)-c_{1} Q^{\prime}+2 c_{2} J Q, \\
& Q_{t}=\frac{v_{0}}{4}\left(Q^{\prime \prime \prime}-6 Q^{2} Q^{\prime}\right)-c_{1} J\left(Q^{\prime \prime}-2 Q^{3}\right)-c_{2} Q^{\prime}+2 c_{3} J Q, \\
& \quad \text { etc. }
\end{aligned}
$$

where $v_{0}$ and $c_{1}, c_{2}, \ldots$ are arbitrary integration constants. Upon rescaling the $t$ variable one may choose $v_{0}=1$. In terms of the AKNS pair $(p, q)$ the homogeneous versions (that is, $c_{1}=c_{2}=\cdots=0$ ) of these equations read

$$
\begin{aligned}
& \left(\begin{array}{l}
p_{t} \\
q_{t}
\end{array}\right)=-v_{0}\left(\begin{array}{c}
p_{x} \\
q_{x}
\end{array}\right), \\
& \left(\begin{array}{c}
p_{t} \\
q_{t}
\end{array}\right)=\frac{i v_{0}}{2}\left(\begin{array}{c}
p_{x x}-2 p^{2} q \\
-q_{x x}+2 p q^{2}
\end{array}\right), \\
& \left(\begin{array}{c}
p_{t} \\
q_{t}
\end{array}\right)=\frac{i v_{0}}{4}\left(\begin{array}{c}
p_{x x x}-6 p q p_{x} \\
-q_{x x x}+6 p q q_{x}
\end{array}\right),
\end{aligned}
$$

etc.

We also mention an interesting scale invariance of the AKNS equations. Suppose $Q$ satisfies one of the AKNS equations, that is, $\operatorname{AKNS}_{n}(Q)=0$. Suppose $a \neq 0$ and

$$
A=\left(\begin{array}{cc}
a & 0 \\
0 & a^{-1}
\end{array}\right) .
$$

Then $\breve{Q}=A Q A$ also satisfies $\operatorname{AKNS}_{n}(\breve{Q})=0$. We omit the straightforward proof which can be found, for instance, in [87].

In the particular case of the nonlinear Schrödinger (NS) hierarchy, where $p(x, t)=$ $\pm \overline{q(x, t)}$, the matrix $A$ is unimodular, that is, $|a|=1$. 
Note that the KdV hierarchy as well as the modified Korteweg-de Vries (mKdV) hierarchy are contained in the AKNS hierarchy. In fact, setting all integration constants $c_{2 \ell+1}$ equal to zero, the $n^{\text {th }} \mathrm{KdV}$ equation is obtained from the $(2 n)^{\text {th }}$ AKNS system by the constraint $p(x, t)=1$, while the $n^{\text {th }} \mathrm{mKdV}$ equation is obtained from the $(2 n)^{\text {th }}$ AKNS system by the constraint $p(x, t)= \pm q(x, t)$.

Just as in the KdV case one makes the following observation: Suppose a polynomial $V_{n+1}$ whose coefficients are scalar functions and a polynomial $\mathcal{W}_{n+1}$ whose coefficients are $2 \times 2$ matrix-valued functions with zero diagonal are given. Furthermore, assume that $V_{n+1}^{\prime}(z, x) I=\mathcal{W}_{n+1}(z, x) Q(x)+Q(x) \mathcal{W}_{n+1}(z, x)$ and that $2 z \mathcal{W}_{n+1}(z, x)+2 V_{n+1}(z, x) J Q(x)-J \mathcal{W}_{n+1}^{\prime}(z, x)$ is independent of $z$. Then the coefficients of $V_{n+1}(z, x)$ and $\mathcal{W}_{n+1}(z, x)$ define a differential expression $P_{n+1}$ which satisfies (4.1.3).

Next, suppose that $\left[P_{n+1}, L\right]=0$ and, without loss of generality, $v_{0} \neq 0$. Then,

$$
\begin{aligned}
\left(P_{n+1}-K_{n+1}(L)\right)^{2} & =\left(J V_{n+1}(L, x)+\mathcal{W}_{n+1}(L, x)\right)^{2} \\
& =\mathcal{W}_{n+1}(L, x)^{2}-V_{n+1}(L, x)^{2} I \\
& =\sum_{m=0}^{2 n+2} a_{m}(x) L^{m}
\end{aligned}
$$

where

$$
a_{m}(x) I=\sum_{\ell+k=m}\left(W_{n+1-\ell}(x) W_{n+1-k}(x)-v_{n+1-\ell}(x) v_{n+1-k}(x) I\right)
$$

is a multiple of the identity matrix. Moreover, differentiating $\left(J V_{n+1}(z, x)+\right.$ $\left.\mathcal{W}_{n+1}(z, x)\right)^{2}$ with respect to $x$ yields

$$
-2 V_{n+1}(z, x) V_{n+1}^{\prime}(z, x) I+\mathcal{W}_{n+1}(z, x) \mathcal{W}_{n+1}^{\prime}(z, x)+\mathcal{W}_{n+1}^{\prime}(z, x) \mathcal{W}_{n+1}(z, x)=0
$$

using

$$
\begin{aligned}
V_{n+1}^{\prime}(z, x) I & =\mathcal{W}_{n+1}(z, x) Q(x)+Q(x) \mathcal{W}_{n+1}(z, x) \\
\mathcal{W}_{n+1}^{\prime}(z, x) & =2 V_{n+1}(z, x) Q-2 z J \mathcal{W}_{n+1}(z, x) \\
0 & =J \mathcal{W}_{n+1}(z, x)+\mathcal{W}_{n+1}(z, x) J
\end{aligned}
$$

Hence the coefficients $a_{m}(x)$ in (4.1.4) may be interpreted as constant scalars. Since $a_{2 n+2}=-v_{0}^{2}$, one infers $\left(P_{n+1}-K_{n+1}(L)\right)^{2}+R_{2 n+2}(L)=0$, where $R_{2 n+2}$ is a polynomial of degree $2 n+2$ with complex coefficients. Hence, if $\left[P_{n+1}, L\right]=0$, then the pair $\left(P_{n+1}, L\right)$ is associated with a hyperelliptic curve of (arithmetic) genus $n$.

Next assume that $F_{n}: \mathbb{C}^{2} \rightarrow \mathbb{C}_{\infty}$ is a polynomial of degree $n$ in its first variable with scalar meromorphic coefficients. Denote the leading coefficient by $-i q(x)$ and let $p(x)$ be another nonzero meromorphic function. Defining

$$
V_{n+1}(z, x)=\frac{-1}{2 q(x)}\left(F_{n}^{\prime}(z, x)+2 i z F_{n}(z, x)\right)
$$

and

$$
\mathcal{W}_{n+1}(z, x)=\frac{i}{q(x)}\left(\begin{array}{cc}
0 & q(x) F_{n}(z, x) \\
V_{n+1}^{\prime}(z, x)+p(x) F_{n}(z, x) & 0
\end{array}\right)
$$


implies $V_{n+1}^{\prime} I=\mathcal{W}_{n+1} Q+Q \mathcal{W}_{n+1}$ with $Q$ given as in (4.1.1). Moreover, $V_{n+1}^{2} I-$ $\mathcal{W}_{n+1}^{2}=R_{2 n+2}(z, x) I$, where the scalar $R_{2 n+2}(z, x)$ is given by

$$
\begin{aligned}
R_{2 n+2}(z, x)= & \frac{1}{4 q(x)^{2}}\left(F_{n}^{\prime}(z, x)^{2}-2 F_{n}(z, x) F_{n}^{\prime \prime}(z, x)+4\left(p(x) q(x)-z^{2}\right) F_{n}(z, x)^{2}\right) \\
& +\frac{q^{\prime}(x)}{4 q(x)^{3}}\left(2 F_{n}(z, x) F_{n}^{\prime}(z, x)+4 i z F_{n}(z, x)^{2}\right) .
\end{aligned}
$$

If this is constant, then differentiation with respect to $x$ yields

$$
\begin{aligned}
\mathcal{W}_{n+1}(z, x)\left(\mathcal{W}_{n+1}^{\prime}(z, x)\right. & \left.-2 V_{n+1}(z, x) Q(x)\right) \\
& +\left(\mathcal{W}_{n+1}^{\prime}(z, x)-2 V_{n+1}(z, x) Q(x)\right) \mathcal{W}_{n+1}(z, x)=0 .
\end{aligned}
$$

Since $\mathcal{W}_{n+1}^{\prime}(z, x)-2 V_{n+1}(z, x) Q(x)$ has zero diagonal elements, this may be considered, for each fixed $x$, a linear homogeneous equation for the off-diagonal elements of $\mathcal{W}_{n+1}^{\prime}(z, x)-2 V_{n+1}(z, x) Q(x)$. This equation has a one-dimensional space of solutions, in fact, $\mathcal{W}_{n+1}^{\prime}(z, x)-2 V_{n+1}(z, x) Q(x)=r(x) J \mathcal{W}_{n+1}(z, x)$. Comparing the leading coefficients yields $r(x)=-2 z$, and hence we have shown that $\mathcal{W}_{n+1}^{\prime}(z, x)=-2 z J \mathcal{W}_{n+1}(z, x)+2 V_{n+1}(z, x) Q(x)$. Summarizing, the following theorem holds.

Theorem 4.1. If $F_{n}: \mathbb{C}^{2} \rightarrow \mathbb{C}_{\infty}$ is a polynomial of degree $n$ in the first variable and meromorphic in the second, and if the expression $R_{2 n+2}(z, x)$ in (4.1.5) is independent of $x$, then there exists a $2 \times 2$ matrix-valued differential expression $P_{n+1}$ of order $n+1$ with leading coefficient $J^{n+2}$ such that $\left[P_{n+1}, L\right]=0$.

We are now ready to define the term algebro-geometric in the context of the AKNS hierarchy.

Definition 4.2. Suppose $Q$ is a $2 \times 2$ matrix-valued meromorphic function, and let $L$ be the differential expression $L=J d / d x+Q$. Then $Q$ is called an algebrogeometric AKNS potential (or simply algebro-geometric) if $Q$ is a solution of some equation of the stationary AKNS hierarchy.

Equivalently, $Q$ is algebro-geometric if any one of the following two conditions is satisfied:

(1) There exists a differential expression $P_{n+1}$ of order $n+1$ with leading coefficient $J^{n+2}$ such that $\left[P_{n+1}, L\right]=0$.

(2) There exists a function $F_{n}: \mathbb{C}^{2} \rightarrow \mathbb{C}_{\infty}$ which is a polynomial in the first variable, meromorphic in the second, such that the expression $R_{2 n+2}(z, x)$ in (4.1.5) does not depend on $x$.

4.2. Periodic AKNS Potentials. Throughout this section we will assume that $p, q \in C^{2}(\mathbb{R})$ are complex-valued periodic functions of period $\Omega>0$. Matrix-valued solutions $Y$ of the initial value problem $(L Y)(x)=z Y(x)$ and $Y\left(x_{0}\right)=Y_{0}$ are denoted by $\Phi\left(z, \cdot, x_{0}, Y_{0}\right)$, which is also the unique solution of the integral equation

$$
Y(x)=\exp \left(z\left(x-x_{0}\right) J\right) Y_{0}+\int_{x_{0}}^{x} \mathrm{e}^{z\left(x-x^{\prime}\right) J} J Q\left(x^{\prime}\right) Y\left(x^{\prime}\right) d x^{\prime} .
$$

In contrast to the Sturm-Liouville case, the Volterra integral equation (4.2.1) is not suitable to determine the asymptotic behavior of solutions as $z$ tends to 
infinity. To circumvent this difficulty one can follow Marchenko's approach in [152], Section 1.4, to obtain the asymptotic expansion

$$
\begin{aligned}
\Phi\left(z, x_{0}+\Omega, x_{0}, I\right)= & \left(\begin{array}{cc}
\mathrm{e}^{-i z \Omega} & 0 \\
0 & \mathrm{e}^{i z \Omega}
\end{array}\right)+\frac{1}{2 i z}\left(\begin{array}{cc}
\beta \mathrm{e}^{-i z \Omega} & 2 q\left(x_{0}\right) \sin (z \Omega) \\
2 p\left(x_{0}\right) \sin (z \Omega) & -\beta \mathrm{e}^{i z \Omega}
\end{array}\right) \\
& +O\left(\mathrm{e}^{|\operatorname{Im}(z)| \Omega} z^{-2}\right),
\end{aligned}
$$

$$
\beta=\int_{x_{0}}^{x_{0}+\Omega} p(t) q(t) d t
$$

provided $p, q \in C^{2}(\mathbb{R})$. From this result we infer that the entries of $\Phi\left(\cdot, x_{0}+\Omega, x_{0}, I\right)$, which are entire functions, have order of growth equal to one whenever $q\left(x_{0}\right)$ and $p\left(x_{0}\right)$ are nonzero.

As in the scalar case we introduce $\mathcal{T}_{\Omega}(z)$, the restriction of the translation operator $Y \mapsto Y(\cdot+\Omega)$ to the two-dimensional space of solutions $\mathcal{Y}(z)$ of $(L Y)(x)=$ $z Y(x)$. The Floquet multipliers are then determined as solutions of

$$
\rho^{2}-\rho \operatorname{tr}\left(\mathcal{T}_{\Omega}(z)\right)+1=0 .
$$

They are degenerate if and only if $\rho(z)^{2}=1$. The points $z$ where this happens are the (anti-)periodic eigenvalues which we denote by $E_{j}, j \in \mathbb{Z}$. Their algebraic multiplicities are given by $\operatorname{ord}_{E_{j}}\left(\mathcal{T}_{\Omega}^{2}-4\right)$ and are denoted by $p\left(E_{j}\right)$. The asymptotic behavior of the (anti-)periodic eigenvalues may be determined from (4.2.2). It is given by

$$
E_{2 j}, E_{2 j-1}=\frac{j \pi}{\Omega}+O\left(|j|^{-1}\right),
$$

if the eigenvalues are labeled in accordance with their algebraic multiplicity.

The conditional stability set $\mathcal{S}(L)$ of $L$ is again

$$
\mathcal{S}(L)=\left\{z \in \mathbb{C} \mid-2 \leq \operatorname{tr}\left(\mathcal{T}_{\Omega}(z)\right) \leq 2\right\} .
$$

The spectrum of the maximal operator in $L^{2}(\mathbb{R})^{2}$ associated with $L$ coincides with the conditional stability set $\mathcal{S}(L)$ of $L$. One can also prove that the conditional stability set $\mathcal{S}(L)$ consists of a countable number of regular analytic arcs, the spectral bands. At most two spectral bands extend to infinity and at most finitely many spectral bands are closed arcs. The point $z$ is a band edge, that is, an endpoint of a spectral band, if and only if $\left(\operatorname{tr}\left(\mathcal{T}_{\Omega}(z)\right)\right)^{2}-4$ has a zero of odd order. For additional results on nonself-adjoint periodic Dirac-type operators, see Tkachenko [213], [215], [216].

The boundary value problem defined by the differential expression $L$ and the requirement that the first component of a solution vanishes at $x_{0}$ and $x_{0}+\Omega$ will (somewhat artificially but in close analogy to the KdV case) be called the Dirichlet problem for the interval $\left[x_{0}, x_{0}+\Omega\right]$. The Dirichlet eigenvalues and their algebraic multiplicities are given as the zeros and their multiplicities of the function

$$
g\left(z, x_{0}\right)=(1,0) \Phi\left(z, x_{0}+\Omega, x_{0}, I\right)(0,1)^{t},
$$

that is, the entry in the upper right corner of $\Phi\left(z, x_{0}+\Omega, x_{0}, I\right)$. The algebraic multiplicity of $z$ as a Dirichlet eigenvalue $\mu(x)$ is denoted by $d(z, x)$. The quantities

$$
d_{i}(z)=\min \{d(z, x) \mid x \in \mathbb{R}\} \text { and } d_{m}(z, x)=d(z, x)-d_{i}(z)
$$

will be called the immovable part and the movable part of the algebraic multiplicity $d(z, x)$, respectively. The sum $\sum_{z \in \mathbb{C}} d_{m}(z, x)$, which is independent of $x$, is 
called the number of movable Dirichlet eigenvalues. Asymptotically, the Dirichlet eigenvalues are distributed according to

$$
\mu_{j}\left(x_{0}\right)=\frac{j \pi}{\Omega}+O\left(|j|^{-1}\right), \quad j \in \mathbb{Z} .
$$

If $q(x) \neq 0$, the function $g(\cdot, x)$ is an entire function with order of growth equal to one. Hadamard's factorization theorem then implies $g(z, x)=F(z, x) D(z)$, where $F$ comprises the factors depending on $x$, while $D$ contains the factors independent of $x$. Of course, $\operatorname{ord}_{z}(F(\cdot, x))=d_{m}(z, x)$ and $\operatorname{ord}_{z}(D)=d_{i}(z)$.

We now turn to the $x$-dependence of the function $g$. The following is the analog of equation (3.1.2) and is obtained by a straightforward computation. The function $F(z, \cdot)$ satisfies the differential equation

$$
\begin{aligned}
& q(x)\left(F^{\prime}(z, x)^{2}-2 F(z, x) F^{\prime \prime}(z, x)+4\left(p(x) q(x)-z^{2}\right) F(z, x)^{2}\right) \\
& +q^{\prime}(x)\left(2 F(z, x) F^{\prime}(z, x)+4 i z F(z, x)^{2}\right)=q(x)^{3}\left(\left(\operatorname{tr}\left(\mathcal{T}_{\Omega}(z)\right)\right)^{2}-4\right) / D(z)^{2} .
\end{aligned}
$$

Just as in the KdV case we get the following theorem.

Theorem 4.3. There exists an entire function $\underline{R}$ such that $\left(\operatorname{tr}\left(\mathcal{T}_{\Omega}(z)\right)\right)^{2}-4=$ $\underline{R}(z)[0] D(z)^{2}$. In particular, $p(z)-2 d_{i}(z) \geq 0$ for every $z \in \mathbb{C}$.

We define the Floquet deficiency of $Q$ (or $L)$ as $\operatorname{def}(Q)=\operatorname{deg}(\underline{R}) \in \mathbb{N}_{0} \cup\{\infty\}$. Equation (4.2.3), Theorem 4.1, and the asymptotic distribution of (anti-)periodic and Dirichlet eigenvalues allow one to prove the following central result.

Theorem 4.4. The following statements are equivalent:

(1) $\operatorname{def}(Q)=2 n+2$.

(2) There are $n$ movable Dirichlet eigenvalues.

(3) There exists a $2 \times 2$ matrix-valued differential expression $P_{n+1}$ of order $n+1$ and leading coefficient $J^{n+2}$ which commutes with $L$. The number $n+1$ is the smallest integer with that property. The differential expression $P_{n+1}$ satisfies $P_{n+1}^{2}=R_{2 n+2}(L)=\prod_{z \in \mathbb{C}}(L-z)^{p(z)-2 d_{i}(z)}$ and hence $Q$ is algebro-geometric.

We remark that in (3) $R_{2 n+2}$ is a constant multiple of $\underline{R}$.

4.3. Necessary Conditions for an AKNS Potential to Be Algebro-Geometric. In this section we will prove the following result.

Theorem 4.5. Suppose $Q$ is an algebro-geometric AKNS potential. Then $L Y=$ $J Y^{\prime}+Q Y=z y$ has a meromorphic fundamental system of solutions with respect to $x$ for all values of the spectral parameter $z \in \mathbb{C}$.

A proof of this theorem along the lines of the proof of Theorem 3.10 fails since one can only show that the exponents of a singularity are half integers rather than integers. However, such reasoning can be used to prove the following result.

Theorem 4.6. Suppose $Q$ is a meromorphic potential coefficient of $L$. Then the equation $(L Y)(x)=z Y(x)$ has a fundamental system of solutions meromorphic with respect to $x$ for all values of the spectral parameter $z \in \mathbb{C}$ whenever this holds for infinitely many values of $z$.

The rest of this section is devoted to a proof of Theorem 4.5. Suppose $Q$ is an algebro-geometric AKNS potential. According to the characterizations following Definition 4.2 , there is a function $F_{n}: \mathbb{C}^{2} \rightarrow \mathbb{C}_{\infty}$ which is a polynomial of degree $n$ in 
the first variable, meromorphic in the second, such that the expression $R_{2 n+2}(z, x)$ in (4.1.5) does not depend on $x$. With the aid of $F_{n}$ we define (as above)

$$
\begin{aligned}
V_{n+1}(z, x) & =\frac{-1}{2 q(x)}\left(F_{n}^{\prime}(z, x)+2 i z F_{n}(z, x)\right), \\
\mathcal{W}_{n+1}(z, x) & =\frac{i}{q(x)}\left(\begin{array}{cc}
0 & q(x) F_{n}(z, x) \\
V_{n+1}^{\prime}(z, x)+p(x) F_{n}(z, x) & 0
\end{array}\right),
\end{aligned}
$$

and

$$
P_{n+1}=J V_{n+1}(L, x)+\mathcal{W}_{n+1}(L, x) .
$$

The pair $\left(P_{n+1}, L\right)$ is associated with the hyperelliptic curve

$$
\mathcal{K}_{n}=\left\{(z, w): w^{2}+R_{2 n+2}(z)=0\right\}
$$

of arithmetic genus $n$, where

$$
R_{2 n+2}(z) I=V_{n+1}(z, x)^{2} I-\mathcal{W}_{n+1}(z, x)^{2}=\prod_{m=1}^{2 n+2}\left(z-E_{m}\right) I .
$$

We emphasize that the points $E_{m}$ are not necessarily distinct.

If $\Psi=\left(\psi_{1}, \psi_{2}\right)^{t}$ is a common solution of $L \Psi=z \Psi$ and $P_{n+1} \Psi=w \Psi$, then, considering the first component of $P_{n+1} \Psi$, we find

$$
\begin{aligned}
w \psi_{1} & =\left(P_{n+1} \Psi\right)_{1}=\left[\left(J V_{n+1}(z, x)+\mathcal{W}_{n+1}(z, x)\right) \Psi\right]_{1}=i V_{n+1} \psi_{1}+\mathcal{W}_{n+1,1,2} \psi_{2} \\
& =\left(i V_{n+1}+\mathcal{W}_{n+1,1,2} \phi\right) \psi_{1},
\end{aligned}
$$

defining $\phi=\psi_{2} / \psi_{1}$. We now reverse this process and define the meromorphic function $\phi$ on $\mathcal{K}_{n} \times \mathbb{C}$ by

$$
\phi((z, w), x)=\frac{w-i V_{n+1}(z, x)}{\mathcal{W}_{n+1,1,2}(z, x)}=\frac{\mathcal{W}_{n+1,2,1}}{w+i V_{n+1}(z, x)} .
$$

We remark that $\phi$ can be extended to a meromorphic function on the compactification (projective closure) of the affine curve $\mathcal{K}_{n}$. This compactification is obtained by joining two points (the points at infinity) to $\mathcal{K}_{n}$.

Next we define

$$
\begin{aligned}
& \psi_{1}\left((z, w), x, x_{0}\right)=\exp \left(\int_{x_{0}}^{x}\left(-i z+q\left(x^{\prime}\right) \phi\left((z, w), x^{\prime}\right)\right) d x^{\prime}\right), \\
& \psi_{2}\left((z, w), x, x_{0}\right)=\phi((z, w), x) \psi_{1}\left((z, w), x, x_{0}\right)
\end{aligned}
$$

where the simple Jordan arc from $x_{0}$ to $x$ in (4.3.1) avoids poles of $q$ and $\phi$. One verifies with the help of $V_{n+1}^{\prime} I=\mathcal{W}_{n+1} Q+Q \mathcal{W}_{n+1}$ and $\mathcal{W}_{n+1}^{\prime}=2 V_{n+1} Q-2 z J \mathcal{W}_{n+1}$ that

$$
\phi^{\prime}((z, w), x)=p(x)-q(x) \phi((z, w), x)^{2}+2 i z \phi((z, w), x) .
$$

From this one obtains that

$$
\Psi\left((z, w), x, x_{0}\right)=\left(\begin{array}{l}
\psi_{1}\left((z, w), x, x_{0}\right) \\
\psi_{2}\left((z, w), x, x_{0}\right)
\end{array}\right)
$$

is a common solution of $L \Psi=z \Psi$ and $P_{n+1} \Psi=w \Psi$.

Let $\mu_{1}\left(x_{0}\right), \ldots, \mu_{n}\left(x_{0}\right)$ denote the zeros of $F_{n}\left(\cdot, x_{0}\right)$. One then observes that the two branches $\Psi_{ \pm}\left(z, \cdot, x_{0}\right)=\Psi\left((z, \pm w), \cdot, x_{0}\right)$ of the function $\Psi\left((z, w), \cdot, x_{0}\right)$ 
represent a fundamental system of solutions of $L y=z y$ for all complex numbers $z$ different from $E_{1}, \ldots, E_{2 n+2}, \mu_{1}\left(x_{0}\right), \ldots, \mu_{n}\left(x_{0}\right)$, since

$$
W\left(\Psi_{-}\left(z, x, x_{0}\right), \Psi_{+}\left(z, x, x_{0}\right)\right)=\frac{2 w}{\mathcal{W}_{n+1,1,2}\left(z, x_{0}\right)}=\frac{-2 i w}{F_{n}\left(z, x_{0}\right)},
$$

where $W(f, g)$ denotes the determinant of the two columns $f$ and $g$.

In the special case where $\mathcal{K}_{n}$ is nonsingular, that is, when the points $E_{m}$ are pairwise distinct, the explicit representation of $\Psi\left((z, w), x, x_{0}\right)$ in terms of the Riemann theta function associated with $\mathcal{K}_{n}$ immediately proves that $\Psi_{ \pm}\left(z, x, x_{0}\right)$ are meromorphic with respect to $x \in \mathbb{C}$ for all $z \in \mathbb{C} \backslash\left\{E_{1}, \ldots, E_{2 n+2}, \mu_{1}\left(x_{0}\right), \ldots, \mu_{n}\left(x_{0}\right)\right\}$. A detailed account of this theta function representation can be found, for instance, in Theorem 3.5 of [87]. In the following we demonstrate how to use gauge transformations to reduce the case of singular curves $\mathcal{K}_{n}$ to nonsingular ones.

Let $Q$ be meromorphic on $\mathbb{C}$ and introduce $A(z, x)=(Q(x)+z I) J$ which turns $L Y=z Y$ into $Y^{\prime}+A Y=0$. Then consider the gauge transformation

$$
\tilde{\Psi}(z, x)=\Gamma(z, x) \Psi(z, x) \text {. }
$$

If

$$
\tilde{A}(z, x)=\Gamma(z, x) A(z, x) \Gamma(z, x)^{-1}-\Gamma^{\prime}(z, x) \Gamma(z, x)^{-1},
$$

then $\tilde{A}(z, x)=(\tilde{Q}(x)+z I) J$, where $\tilde{Q}$ has zero diagonal elements. Moreover, $\tilde{\Psi}^{\prime}+\tilde{A} \tilde{\Psi}=0$, that is, $(J d / d x+\tilde{Q}) \tilde{\Psi}=z \tilde{\Psi}$. Next we make the following explicit choice suggested by Konopelchenko [127]. Let $\tilde{z} \in \mathbb{C}$ be fixed, $\Psi^{(0)}(\tilde{z}, \cdot)=$ $\left(\psi_{1}^{(0)}(\tilde{z}, \cdot), \psi_{2}^{(0)}(\tilde{z}, \cdot)\right)^{t}$ be any solution of $L y=\tilde{z} y$, and introduce

$$
\phi^{(0)}(\tilde{z}, x)=\psi_{2}^{(0)}(\tilde{z}, x) / \psi_{1}^{(0)}(\tilde{z}, x) .
$$

Then one defines

$$
\Gamma(z, x)=\frac{1}{2}\left(\begin{array}{cc}
2(z-\tilde{z})-i q(x) \phi^{(0)}(\tilde{z}, x) & i q(x) \\
i \phi^{(0)}(\tilde{z}, x) & -i
\end{array}\right)
$$

for $z \in \mathbb{C} \backslash\{\tilde{z}\}$.

The upper right entry $G_{1,2}\left(z, x, x^{\prime}\right)$ of the Green's matrix of $L$ is given by

$$
G_{1,2}\left(z, x, x^{\prime}\right)=i \frac{\psi_{+, 1}\left(z, x, x_{0}\right) \psi_{-, 1}\left(z, x^{\prime}, x_{0}\right)}{W\left(\Psi_{-}\left(z, \cdot, x_{0}\right), \Psi_{+}\left(z, \cdot, x_{0}\right)\right)}, \quad x \geq x^{\prime} .
$$

We want to evaluate $G_{1,2}\left(z, x, x^{\prime}\right)$ on its diagonal (i.e., where $\left.x=x^{\prime}\right)$. Since $\psi_{+, 1}\left(z, x_{0}, x_{0}\right) \psi_{-, 1}\left(z, x_{0}, x_{0}\right)=1$ we obtain from (4.3.1) and (4.3.3)

$$
G_{1,2}(z, x, x)=i \frac{\mathcal{W}_{n+1,1,2}(z, x)}{2 w}=\frac{-F_{n}(z, x)}{2 w} .
$$

Next note that

$$
W\left(\tilde{\Psi}_{-}(z, \cdot), \tilde{\Psi}_{+}(z, \cdot)\right)=\operatorname{det}(\Gamma(z, x)) W\left(\Psi_{-}(z, \cdot), \Psi_{+}(z, \cdot)\right)
$$

and that $\operatorname{det}(\Gamma(z, x))=-i(z-\tilde{z}) / 2$. With the help of this fact and some computations one finds that the upper right entry $\tilde{G}_{1,2}(z, x, x)$ of the Green's matrix of $J d / d x+\tilde{Q}$ is

$$
\tilde{G}_{1,2}(z, x, x)=i \frac{\tilde{\psi}_{+, 1}(z, x) \tilde{\psi}_{-, 1}(z, x)}{W\left(\tilde{\Psi}_{-}(z, \cdot), \tilde{\Psi}_{+}(z, \cdot)\right)}=\frac{-\tilde{F}_{n+1}(z, x)}{2(z-\tilde{z}) w},
$$


where $\tilde{F}_{n+1}(\cdot, x)$ is a polynomial of degree $n+1$ with leading coefficient $-i \tilde{q}(x)$. Moreover, $\tilde{F}_{n+1}(z, x)$ satisfies

$$
\begin{aligned}
& \tilde{q}\left(2 \tilde{F}_{n+1} \tilde{F}_{n+1}^{\prime \prime}-\tilde{F}_{n+1}^{\prime 2}+4\left(z^{2}-\tilde{p} \tilde{q}\right) \tilde{F}_{n+1}^{2}\right)-\tilde{q}^{\prime}\left(2 \tilde{F}_{n+1} \tilde{F}_{n+1}^{\prime}+4 i z \tilde{F}_{n+1}^{2}\right) \\
= & -4 \tilde{q}^{3}(z-\tilde{z})^{2} R_{2 n+2}(z) .
\end{aligned}
$$

Hence $\tilde{Q}$ is an algebro-geometric AKNS potential.

Now suppose that $(\tilde{z}, 0)$ is a singular point of $\mathcal{K}_{n}$, that is, that $\tilde{z}$ is a zero of $R_{2 n+2}$ of order $r \geq 2$. Choose

$$
\phi^{(0)}(\tilde{z}, x)=\frac{-i V_{n+1}(\tilde{z}, x)}{\mathcal{W}_{n+1,1,2}(\tilde{z}, x)} .
$$

Then one may show that $\tilde{F}_{n+1}(\cdot, x)$ has a zero of order at least 2 at $\tilde{z}$, that is,

$$
\tilde{F}_{n+1}(z, x)=(z-\tilde{z})^{s} \hat{F}_{\hat{n}}(z, x)
$$

for some $s \geq 2$ and some polynomial $\hat{F}_{\hat{n}}(\cdot, x)$ of degree $\hat{n}=n+1-s$. From (4.3.4) one obtains

$$
\tilde{q}\left(2 \hat{F}_{\hat{n}} \hat{F}_{\hat{n}}^{\prime \prime}-\hat{F}_{\hat{n}}^{\prime 2}+4\left(z^{2}-\tilde{p} \tilde{q}\right) \hat{F}_{\hat{n}}^{2}\right)-\tilde{q}^{\prime}\left(2 \hat{F}_{\hat{n}} \hat{F}_{\hat{n}}^{\prime}+4 i z \hat{F}_{\hat{n}}^{2}\right)=-4 \tilde{q}^{3} \hat{R}_{2 n-2 s+4}(z),
$$

where

$$
\hat{R}_{2 n-2 s+4}(z)=(z-\tilde{z})^{2-2 s} R_{2 n+2}(z)
$$

is a polynomial in $z$ of degree $2 n-2 s+4 \in(0,2 n+2)$. This proves that $\tilde{Q}$ is associated with the curve

$$
\mathcal{K}_{\hat{n}}=\left\{(z, w): w^{2}+(z-\tilde{z})^{2-2 s} R_{2 n+2}(z)=0\right\} .
$$

Our choice of $\phi^{(0)}(\tilde{z}, x)$ led to a curve $\mathcal{K}_{\hat{n}}$ which is less singular at $(\tilde{z}, 0)$ than $\mathcal{K}_{n}$ without changing the local structure of the curve elsewhere. By iterating this procedure one ends up with a curve which is nonsingular at $(\tilde{z}, 0)$. Repeating the procedure for each singular point of $\mathcal{K}_{n}$ then results in a nonsingular curve and a corresponding Baker-Akhiezer function $\hat{\Psi}\left((z, w), x, x_{0}\right)$ meromorphic with respect to $x \in \mathbb{C}$ using its standard theta function representation (see, e.g., [87]). Since $\phi^{(0)}=-i V_{n+1} / \mathcal{W}_{n+1,1,2}$ is meromorphic, the gauge transformations and their inverses map meromorphic functions to meromorphic functions. Combining these results proves Theorem 4.5 .

A systematic account of the effect of Darboux-type transformations on hyperelliptic curves in connection with the KdV, AKNS, and Toda hierarchies will be presented in [85].

\subsection{Sufficient Conditions for an AKNS Potential to Be Algebro-Geome-} tric - A Characterization of Elliptic Stationary AKNS Solutions.

Definition 4.7. Let $p, q$ be elliptic functions with a common period lattice. Then $Q=\left(\begin{array}{cc}0 & -i q \\ i p & 0\end{array}\right)$ is called a Picard-AKNS potential if the equation $J \psi^{\prime}(x)+Q(x) \psi(x)$ $=z \psi(x)$ has a meromorphic fundamental system of solutions with respect to $x$ for all values of the spectral parameter $z \in \mathbb{C}$.

We note that according to Theorem 4.6 it is sufficient to show the existence of a meromorphic fundamental system for infinitely many values of $z$ in order to prove that $Q$ is a Picard-AKNS potential.

Just as in the KdV case the following theorem characterizes all elliptic algebrogeometric AKNS potentials. 
Theorem 4.8. Let $Q=\left(\begin{array}{cc}0 & -i q \\ i p & 0\end{array}\right)$ with $p, q$ elliptic functions with a common period lattice. Then $Q$ is an elliptic algebro-geometric AKNS potential if and only if it is a Picard-AKNS potential.

Sketch of proof. The necessity of the criterion is the content of Theorem 4.5. The sufficiency follows from Picard's Theorem 2.7 and Theorem 4.4 in the same way as in the $\mathrm{KdV}$ case.

The transformation

$$
\tilde{\Psi}(x)=\left(\begin{array}{cc}
\mathrm{e}^{a x+b} & 0 \\
0 & \mathrm{e}^{-(a x+b)}
\end{array}\right) \Psi(x)
$$

allows one to prove the following corollary, which slightly extends the class of algebro-geometric AKNS potentials $Q$ considered thus far. Such cases have recently been considered by Smirnov [199].

Corollary 4.9. Suppose

$$
Q(x)=\left(\begin{array}{cc}
0 & -i q(x) \mathrm{e}^{-2(a x+b)} \\
i p(x) \mathrm{e}^{2(a x+b)} & 0
\end{array}\right),
$$

where $a, b \in \mathbb{C}$ and $p, q$ are elliptic functions with a common period lattice. Then $Q$ is an algebro-geometric AKNS potential if $J \Psi^{\prime}(x)+Q(x) \Psi(x)=z \Psi(x)$ has a meromorphic fundamental system of solutions with respect to $x$ for all values of the spectral parameter $z \in \mathbb{C}$.

4.5. Examples. With the exception of the studies by Christiansen, Eilbeck, Enol'skii, and Kostov [39] and Smirnov [199], [202], [203], not too many examples of elliptic solutions $(p, q)$ of the AKNS hierarchy associated with higher (arithmetic) genus curves of the type $w^{2}+R_{2 n+2}(z)=0$ have been worked out in detail. The genus $n=1$ case has been considered, for example, by Its [120] and Pavlov [176]. Moreover, examples for low genus $n$ for special cases such as the nonlinear Schrödinger and mKdV equation are considered, for instance, in [8], [14], [147], [161], [155], [171], [200]. Examples related to equations of the sine-Gordon-type are discussed in [196], [204], [205]. The following examples in (4.5.1), (4.5.2), (4.5.3), (4.5.4) are algebro-geometric AKNS potentials as can be proved using the Frobenius method. For details on this procedure see [98].

$$
\begin{aligned}
& Q(x)=i n\left(\zeta(x)-\zeta\left(x-\omega_{2}\right)-\zeta\left(\omega_{2}\right)\right)\left(\begin{array}{cc}
0 & -1 \\
1 & 0
\end{array}\right), \quad n \in \mathbb{N}, \\
& Q(x)=\left(\begin{array}{cc}
0 & -i n(n+1) \wp(x) \\
i & 0
\end{array}\right), \quad n \in \mathbb{N}, \\
& Q(x)=\left(\begin{array}{cc}
0 & i \wp^{\prime}\left(x-\omega_{2}\right) /\left(2 e_{1}\right) \\
-3 i \wp^{\prime}(x) /\left(2 e_{1}\right) & 0
\end{array}\right), \quad e_{2}=0, \\
& Q(x)=\left(\begin{array}{cc}
0 & i \wp\left(x-\omega_{2}\right) / e_{1}^{2} \\
2 i\left(\wp^{\prime \prime}(x)-e_{1}^{2}\right) / 3 & 0
\end{array}\right), \quad e_{2}=0 .
\end{aligned}
$$

Incidentally, if $p=1$, as in Example (4.5.2), then $J \Psi^{\prime}+Q \Psi=z \Psi$ is equivalent to the scalar equation $\psi_{2}^{\prime \prime}-q \psi_{2}=-z^{2} \psi_{2}$, where $\Psi=\left(\psi_{1}, \psi_{2}\right)^{t}$ and $\psi_{1}=\psi_{2}^{\prime}-i z \psi_{2}$. Therefore, if $-q$ is an elliptic algebro-geometric potential of the KdV hierarchy, 
then, by Theorem 3.12, $\psi_{2}$ is meromorphic for all values of $z$. Hence $\Psi$ is meromorphic for all values of $z$ and therefore $Q$ is a Picard-AKNS and hence an algebrogeometric AKNS potential. Conversely, if $Q$ is an algebro-geometric AKNS potential with $p=1$, then $-q$ is an algebro-geometric potential of the KdV hierarchy. In particular, $q(x)=n(n+1) \wp(x)$ is again the class of Lamé potentials associated with the KdV hierarchy and hence a special case of the material discussed in Section 3.2.

\section{REFERENCES}

1. M. J. Ablowitz and P.A.Clarkson, Solitons, Nonlinear Evolution Equations and Inverse Scattering, Cambridge Univ. Press, Cambridge, 1991. MR 93g:35108

2. M. J. Ablowitz, D. J. Kaup, A. C. Newell, and H. Segur, The inverse scattering transform Fourier analysis for nonlinear problems, Stud. Appl. Math. 53 (1974), 249-315. MR 56:9108

3. M. Abramowitz and I. A. Stegun, Handbook of Mathematical Functions, Dover, New York, 1972. MR 94b:00012

4. M. Adler and J. Moser, On a class of polynomials connected with the Korteweg-de Vries equation, Commun. Math. Phys. 61 (1978), 1-30. MR 58:18554

5. H. Airault, H. P. McKean, and J. Moser, Rational and elliptic solutions of the KortewegdeVries equation and a related many-body problem, Commun. Pure Appl. Math. 30 (1977), 95-148. MR 58:31214

6. N. I. Akhiezer, On the spectral theory of Lamé's equation, Istor.-Mat. Issled 23 (1978), 77-86, 357. (Russian). MR 82h:34029

7. 1990. MR 91k:33016

8. G. L. Alfimov, A. R. Its, and N. E. Kulagin, Modulation instability of solutions of the nonlinear Schrödinger equation, Theoret. Math. Phys. 84 (1990), 787-793. MR 91h:35294

9. P. É. Appell, Sur la transformation des équations différentielles linéaires, Comptes Rendus 91 (1880), 211-214.

10. F. M. Arscott, Periodic Differential Equations, MacMillan, New York, 1964. MR 30:4006

11. N. Asano and Y. Kato, Algebraic and Spectral Methods for Nonlinear Wave Equations, Longman, New York, 1990. MR 92d:35001

12. O. Babelon and M. Talon, The symplectic structure of the spin Calogero model, Phys. Lett. A 236 (1997), 462-468. CMP 98:06

13. M. V. Babich, A. I. Bobenko, and V. B. Matveev, Reductions of Riemann theta-functions of genus $g$ to theta-functions of lower genus, and symmetries of algebraic curves, Sov. Math. Dokl. 28 (1983), 304-308. MR 85f:14046

14. - Solutions of nonlinear equations integrable in Jacobi theta functions by the method of the inverse problem, and symmetries of algebraic curves, Math. USSR Izv. 26 (1986), 479-496. MR 87d:58069

15. H. F. Baker, Note on the foregoing paper, "Commutative ordinary differential operators," by J. L. Burchnall and J. W. Chaundy, Proc. Roy. Soc. London A 118 (1928), 584-593.

16. E. D. Belokolos, A. I. Bobenko, V. Z. Enol'skii, A. R. Its, and V. B. Matveev, AlgebroGeometric Approach to Nonlinear Integrable Equations, Springer, Berlin, 1994.

17. E. D. Belokolos, A. I. Bobenko, V. B. Matveev, and V. Z. Enol'skii, Algebraic-geometric principles of superposition of finite-zone solutions of integrable non-linear equations, Russian Math. Surv. 41:2 (1986), 1-49. MR 87i:58078

18. E. D. Belokolos and V. Z. Enol'skii, Verdier elliptic solitons and the Weierstrass theory of reduction, Funct. Anal. Appl. 23 (1989), 46-47. MR 90h:14059

19. _ Isospectral deformations of elliptic potentials, Russ. Math. Surv. 44:5 (1989), 191193. MR 91c:58046

20. _ Reduction of theta functions and elliptic finite-gap potentials, Acta Appl. Math. 36 (1994), 87-117. MR 95j:35205

21. D. Bennequin, Hommage à Jean-Louis Verdier: au jardin des systèmes intégrables, in Integrable Systems: The Verdier Memorial Conference (ed. by O. Babelon, P. Cartier, and Y. Kosmann-Schwarzbach), Birkhäuser, Boston, 1993, 1-36. MR 95g:01020

22. G. D. Birkhoff, Existence and oscillation theorem for a certain boundary value problem, Trans. Amer. Math. Soc. 10 (1909), 259-270. 
23. B. Birnir, Complex Hill's equation and the complex periodic Korteweg-de Vries equations, Commun. Pure Appl. Math. 39 (1986), 1-49. MR 87f:58061

24. - Singularities of the complex Korteweg-de Vries flows, Commun. Pure Appl. Math. 39 (1986), 283-305. MR 87k:58109

25. , An example of blow-up, for the complex KdV equation and existence beyond blow-up, SIAM J. Appl. Math. 47 (1987), 710-725. MR 88i:35139

26. G. Borg, Eine Umkehrung der Sturm-Liouvilleschen Eigenwertaufgabe, Acta Math. 78 (1946), 1-96. MR 7:382d

27. V. M. Buchstaber, V. Z. Enol'skii, and D. V. Leykin, Hyperelliptic Kleinian functions and applications, in Solitons, Geometry, and Topology: On the Crossroad, V. M. Buchstaber and S. P. Novikov Eds.), Amer. Math. Soc. Transl. (2), 179 (1997), 1-33. MR 98b:14029

28. __ Kleinian functions, hyperelliptic Jacobians and applications, to appear in Revs. in Mathematics and Mathematical Physics, Vol. 10, S. Novikov and I. Krichever (eds.), Gordon \& Breach, pp. 1-115.

29. J. L. Burchnall and T. W. Chaundy, Commutative ordinary differential operators, Proc. London Math. Soc. Ser. 221 (1923), 420-440.

30. - Commutative ordinary differential operators, Proc. Roy. Soc. London A 118 (1928), $557-583$.

31. Commutative ordinary differential operators. II.-The identity $P^{n}=Q^{m}$, Proc. Roy. Soc. London A134 (1932), 471-485.

32. H. Burkhardt, Elliptische Functionen, 2nd ed., Verlag von Veit, Leipzig, 1906.

33. M. Buys and A. Finkel, The inverse periodic problem for Hill's equation with a finite-gap potential, J. Diff. Eqs. 55 (1984), 257-275. MR 86a:34052

34. F. Calogero, Exactly solvable one-dimensional many-body problems, Lett. Nuovo Cim. 13 (1975), 411-416. MR 52:9728

35. Motion of poles and zeros of special solutions of nonlinear and linear partial differential equations and related "solvable" many-body problems, Nuovo Cim. 43B (1978), 177-241. MR 80a:58023

36. R. C. Carlson and K. R. Goodearl, Commutants of ordinary differential operators, J. Diff. Eqs. 35 (1980), 339-365. MR 81g:12025

37. K. Chandrasekharan, Elliptic Functions, Springer, Berlin, 1985. MR 87e:11058

38. D. V. Choodnovsky and G. V. Choodnovsky, Pole expansions of nonlinear partial differential equations, Nuovo Cim. 40B (1977), 339-353. MR 56:6722

39. P. L. Christiansen, J. C. Eilbeck, V. Z. Enol'skii, and N. A. Kostov, Quasi-periodic solutions of the coupled nonlinear Schrödinger equations, Proc. Roy. Soc. London A 451 (1995), 685-700. MR 96k:34081

40. D. V. Chudnovsky, Meromorphic solutions of nonlinear partial differential equations and many-particle completely integrable systems, J. Math. Phys. 20 (1979), 2416-2422. MR 81h:35043

41. D. V. Chudnovsky and G. V. Chudnovsky, Appendix I: Travaux de J. Drach (1919), Classical and Quantum Models and Arithmetic Problems (ed. by D. V. Chudnovsky and G. V. Chudnovsky), Marcel Dekker, New York, 1984, 445-453. MR 86i:34011

42. E. A. Coddington and N. Levinson, Theory of Ordinary Differential Equations, Krieger, Malabar, 1985. MR 16:1022b

43. E. Colombo, G. P. Pirola, and E. Previato, Density of elliptic solitons, J. reine angew. Math. 451 (1994), 161-169. MR 95e:58079

44. L. A. Dickey, Soliton Equations and Hamiltonian Systems, World Scientific, Singapore, 1991. MR 93d:58067

45. R. K. Dodd, J. C. Eilbeck, J. D. Gibbon, and H. C. Morris, Solitons and Nonlinear Wave Equations, Academic Press, London, 1988. MR 84j:35142

46. R. Donagi and E. Markman, Spectral covers, algebraically completely integrable, Hamiltonian systems, and moduli of bundles, in Integrable Systems and Quantum Groups (ed. by R. Donagi, B. Dubrovin, E. Frenkel, and E. Previato), Lecture Notes in Mathematics 1620, Springer, Berlin, 1996, 1-119. MR 97h:14017

47. R. Donagi and E. Witten, Supersymmetric Yang-Mills theory and integrable systems, Nuclear Phys. B 460 (1996), 299-334. MR 97a:58076

48. J. Drach, Sur les groupes complexes de rationalité et sur l'intégration par quadratures, C. R. Acad. Sci. Paris 167 (1918), 743-746. 
49. Détermination des cas de réduction de'léquation différentielle $d^{2} y / d x^{2}=[\phi(x)+h] y$, C. R. Acad. Sci. Paris 168 (1919), 47-50.

50. _ Sur l'intégration par quadratures de'léquation $d^{2} y / d x^{2}=[\phi(x)+h] y$, C. R. Acad. Sci. Paris 168 (1919), 337-340.

51. P. G. Drazin and R. S. Johnson, Solitons: an introduction, Cambridge University Press, Cambridge, 1989. MR 90j:35166

52. B. A. Dubrovin, Periodic problems for the Korteweg-de Vries equation in the class of finitegap potentials, Funct. Anal. Appl. 9, (1975), 215-223. MR 58:6480

53. Completely integrable Hamiltonian systems associated with matrix operators and Abelian varieties, Funct. Anal. Appl. 11 (1977), 265-277. MR 58:31219

54. _ Theta functions and non-linear equations, Russ. Math. Surv. 36:2 (1981), 11-92.

55. __ Matrix finite-zones operators, Revs. Sci. Technology 23 (1983), 20-50. MR 86a:58041

56. B. A. Dubrovin and S. P. Novikov, Periodic and conditionally periodic analogs of the manysoliton solutions of the Korteweg-de Vries equation, Sov. Phys.-JETP 40 (1975), 1058-1063. MR 52:3759

57. M. S. P. Eastham, The Spectral Theory of Periodic Differential Equations, Scottish Academic Press, Edinburgh and London, 1973.

58. J. C. Eilbeck and V. Z. Enol'skii, Elliptic Baker-Akhiezer functions and an application to an integrable dynamical system, J. Math. Phys. 35 (1994), 1192-1201. MR 94m:58104

59. __ Elliptic solutions and blow-up in an integrable Hénon-Heiles system, Proc. Roy. Soc. Edinburgh 124A (1994), 1151-1164. MR 95j:58067

60. V. Z. Enol'skii, On the solutions in elliptic functions of integrable nonlinear equations, Phys. Lett. 96A (1983), 327-330. MR 85e:58064

61. __ On the two-gap Lamé potentials and elliptic solutions of the Kovalevskaja problem connected with them, Phys. Lett. 100A (1984), 463-466. MR 85k:35200

62. __ On solutions in elliptic functions of integrable nonlinear equations associated with two-zone Lamé potentials, Soc. Math. Dokl. 30 (1984), 394-397. MR 86c:35134

63. V. Z. Enol'skii and J. C. Eilbeck, On the two-gap locus for the elliptic Calogero-Moser model, J. Phys. A 28 (1995), 1069-1088. MR 96a:58149

64. V. Z. Enol'skii and N. A. Kostov, On the geometry of elliptic solitons, Acta Appl. Math. 36 (1994), 57-86. MR 95k:14066

65. A. Erdélyi, On Lamé functions, Phil. Mag. (7) 31 (1941), 123-1130. MR 2:285a

66. E. Fermi, J. Pasta, and S. M. Ulam, Studies in nonlinear problems, Technical Report LA-1940, Los Alamos Sci. Lab. Also in: Collected Papers of Enrico Fermi, Vol II, 978-988, University of Chicago Press, 1965.

67. A. Finkel, E. Isaacson and E. Trubowitz, An explicit solution of the inverse periodic problem for Hill's equation, SIAM J. Math. Anal. 18 (1987), 46-53. MR 88d:34037

68. H. Flaschka, On the inverse problem for Hill's operator, Arch. Rat. Mech. Anal. 59 (1975), 293-309. MR 52:8550

69. G. Floquet, Sur la théorie des équations différentielles linéaires, Ann. Sci. École Norm. Sup. 8 (1879), suppl., 1-132.

70. _ Sur les équations différentielles linéaires à coefficients périodiques, C. R. Acad. Sci. Paris 91 (1880), 880-882.

71. __ Sur les équations différentielles linéaires à coefficients périodiques, Ann. Sci. École Norm. Sup. 12 (1883), 47-88.

72. _ Sur les équations différentielles linéaires à coefficients doublement périodiques, C. R. Acad. Sci. Paris 98 (1884), 38-39, 82-85.

73. __ Sur les équations différentielles linéaires à coefficients doublement périodiques, Ann. Sci. Ecole Norm. Sup. 1 (1884), 181-238.

74. __ Addition a un mémorie sur les équations différentielles linéaires, Ann. Sci. Ecole Norm. Sup. 1 (1884), 405-408.

75. A. R. Forsyth, Theory of Differential Equations, Part III, Vol. 4, Dover, New York, 1959. MR 23:A1079

76. C. S. Gardner, J. M. Greene, M. D. Kruskal, and R. M. Miura, Method for solving the Korteweg-de Vries equation, Phys. Rev. Lett. 19 (1967), 1095-1097.

77. C. S. Gardner, J. M. Greene, M. D. Kruskal, and R. M. Miura, Korteweg-de Vries equation and generalizations. VI. Methods for exact solution, Commun. Pure Appl. Math. 27 (1974), 97-133. MR 49:898 
78. C. S. Gardner and G. K. Morikawa, Similarity in the asymptotic behavior of collision free hydromagnetic waves and water waves, Research Report NYO-9082, Courant Institute, 1960.

79. M. G. Gasymov, Spectral analysis of a class of second-order non-self-adjoint differential operators, Funct. Anal. Appl. 14 (1980), 11-15. MR 81c:47048

80. M. G. Gasymov, Spectral analysis of a class of ordinary differential operators with periodic coefficients, Sov. Math. Dokl. 21 (1980), 718-721. MR 81h:34023

81. L. Gatto and S. Greco, Algebraic curves and differential equations: an introduction, The Curves Seminar at Queen's, Vol. VIII (ed. by A. V. Geramita), Queen's Papers Pure Appl. Math. 88, Queen's Univ., Kingston, Ontario, Canada, 1991, B1-B69. MR 93d:58069

82. I. M. Gel'fand and L. A. Dikii, Asymptotic behaviour of the resolvent of Sturm-Liouville equations and the algebra of the Korteweg-de Vries equations, Russ. Math. Surv. 30:5, (1975) 77-113. MR 58:22746

83. _ Fractional powers of operators and Hamiltonian systems, Funct. Anal. Appl. 10 (1976), 259-272. MR 55:6484

84. (1979), 6-15. MR 80i:58027

85. F. Gesztesy and H. Holden, Darboux-type transformations and hyperelliptic curves, in preparation.

86. - Hierarchies of Soliton Equations and their Algebro-Geometric Solutions, monograph in preparation.

87. F. Gesztesy and R. Ratneseelan, An alternative approach to algebro-geometric solutions of the AKNS hierarchy, Rev. Math. Phys. 10 (1998), 345-391. CMP 98:14

88. F. Gesztesy and B. Simon, The xi function, Acta Math. 176 (1996), 49-71. MR 97e:47078

89. F. Gesztesy, B. Simon, and G. Teschl, Spectral deformations of one-dimensional Schrödinger operators, J. d'Anal. Math. 70 (1996), 267-324. CMP 97:11

90. F. Gesztesy and W. Sticka, On a theorem of Picard, Proc. Amer. Math. Soc. 126 (1998), 1089-1099. CMP 98:06

91. F. Gesztesy and R. Weikard, Spectral deformations and soliton equations, Differential Equations with Applications to Mathematical Physics (ed. by W. F. Ames, E. M. Harrell II, and J. V. Herod), Academic Press, Boston, 1993, 101-139. MR 93m:34138

92. _ Floquet theory revisited, Differential Equations and Mathematical Physics (ed. by I. Knowles), International Press, Boston, 1995, 67-84.

93. _ Lamé potentials and the stationary $(m) K d V$ hierarchy, Math. Nachr. 176 (1995), 73-91. MR 98a:58086

94. $\quad$ Treibich-Verdier potentials and the stationary $(m) K d V$ hierarchy, Math. Z. 219 (1995), 451-476. MR 96e:14030

95. _ On Picard potentials, Diff. Int. Eqs. 8 (1995), 1453-1476. MR 96e:34141

96. - A characterization of elliptic finite-gap potentials, C. R. Acad. Sci. Paris 321 (1995), 837-841. MR 96k:58112

97. MR 97f: 14046

98. _ A characterization of all elliptic algebro-geometric solutions of the AKNS hierarchy, Acta Math. 181 (1998), to appear.

99. - Toward a characterization of elliptic solutions of hierarchies of soliton equations, Contemp. Math., to appear.

100.

101. M. Giertz, M. K. Kwong, and A. Zettl, Commuting linear differential expressions, Proc. Roy. Soc. Edinburgh 87A (1981), 331-347. MR 83d:12011

102. J. Gray, Linear Differential Equations and Group Theory from Riemann to Poincaré, Birkhäuser, Boston, 1986. MR 89d:01041

103. S. Greco and E. Previato, Spectral curves and ruled surfaces: projective models, in The Curves Seminar at Queen's, Vol. VIII (ed. by A. V. Geramita), Queen's Papers Pure Appl. Math. 88, Queen's Univ., Kingston, Ontario, Canada, 1991, F1-F33. MR 93e:58084

104. P. G. Grinevich, Rational solutions for the equation of commutation of differential operators, Funct. Anal. Appl. 16 (1982), 15-19. MR 83f:58040

105. V. Guillemin and A. Uribe, Hardy functions and the inverse spectral method, Commun. PDE 8 (1983), 1455-1474. MR 85h:35197 
106. G.-H. Halphen, Memoire sur la reduction des equations differentielles lineaires aux formes integrales, Mem. pres. l'Acad. Sci., France 28 (1884), 1-300.

107. _ Sur une nouvelle classe d'équations différentielles linéaires intégrables, C. R. Acad. Sci. Paris 101 (1885), 1238-1240.

108. _ Traité des Fonctions Elliptiques, tome 2, Gauthier-Villars, Paris, 1888.

109. G. Hamel, Über die lineare Differentialgleichung zweiter Ordnung mit periodischen Koeffizienten, Math. Ann. 73 (1913), 371-412.

110. O. Haupt, Über lineare homogene Differentialgleichungen 2. Ordnung mit periodischen Koeffizienten, Math. Ann. 79 (1919), 278-285.

111. C. Hermite, Sur quelques applications des fonctions elliptiques, Comptes Rendus 85 (1877), 689-695, 728-732, 821-826.

112. _ Oeuvres, tome 3, Gauthier-Villars, Paris, 1912.

113. G. W. Hill, On the part of the motion of the lunar perigee which is a function of the mean motions of the sun and moon, Acta Math. 8 (1886), 1-36. Reprinted from a paper first published in 1877 .

114. E. Hille, Ordinary Differential Equations in the Complex Domain, Dover, Mineola, N.Y., 1997. MR 97m:34001

115. H. Hochstadt, On the determination of a Hill's equation from its spectrum, Arch. Rat. Mech. Anal. 19 (1965), 353-362. MR 31:6019

116. I. D. Iliev, E. Kh. Khristov, and K. P. Kirchev, Spectral methods in Soliton Equations, Longman, New York, 1994. MR 97e:35129

117. E. L. Ince, Further investigations into the periodic Lamé functions, Proc. Roy. Soc. Edinburgh 60 (1940), 83-99. MR 2:46d

118. _ Ordinary Differential Equations, Dover, New York, 1956. MR 6:65f

119. H. Itoyama and A. Morozov, Integrability and Seiberg-Witten theory curves and periods, Nuclear Phys. B 477 (1996), 855-877. MR 98d:81116

120. A. R. Its, Inversion of hyperelliptic integrals and integration of nonlinear differential equations, Vestnik Leningrad Univ. Math. 9 (1981), 121-129. MR 58:29453

121. A. R. Its and V. Z. Enol'skii, Dynamics of the Calogero-Moser system and the reduction of hyperelliptic integrals to elliptic integrals , Funct. Anal. Appl. 20 (1986), 62-64. MR 87j:14072

122. A. R. Its and V. B. Matveev, Schrödinger operators with finite-gap spectrum and N-soliton solutions of the Korteweg-de Vries equation, Theoret. Math. Phys. 23 (1975), 343-355. MR $57: 18570$

123. K. Iwasaki, Inverse problem for Sturm-Liouville and Hill equations, Ann. Math. Pura Appl. Ser. 4, 149 (1987), 185-206. MR 89d:34053

124. F. Klein, Über den Hermite'schen Fall der Lamé'schen Differentialgleichung, Math. Ann. 40 (1892), 125-129.

125. Q. Kong and A. Zettl, Dependence of eigenvalues of Sturm-Liouville problems on the boundary, J. Diff. Eqs. 126 (1996), 389-407. MR 97c:34176

126. Eigenvalues of regular Sturm-Liouville problems, J. Diff. Eqs. 131 (1996), 1-19. MR 97g:34106

127. B. G. Konopelchenko, Elementary Bäcklund transformations, nonlinear superposition principle and solutions of the integrable equations, Phys. Lett. 87A (1982), 445-448. MR 84a:58049

128. D. J. Korteweg and G. de Vries, On the change of form of long waves advancing in a rectangular canal, and on a new type of long stationary waves, Phil. Mag. 39 (1895), 422443.

129. N. A. Kostov and V. Z. Enol'skii, Spectral characteristics of elliptic solitons, Math. Notes 53 (1993), 287-293. MR 95j:58072

130. S. Kotani, Generalized Floquet theory for stationary Schrödinger operators in one dimension, Chaos, Solitons and Fractals 8 (1997), 1817-1854. CMP 98:03

131. M. Krause, Theorie der doppeltperiodischen Funktionen einer veränderlichen Grösse, Vol. 1, 1895, Vol. 2, 1897, Teubner, Leipzig.

132. I. M. Krichever, Integration of nonlinear equations by the methods of algebraic geometry, Funct. Anal. Appl. 11 (1977), 12-26.

133. _ Methods of algebraic geometry in the theory of non-linear equations, Russ. Math. Surv. 32:6 (1977), 185-213. 
134. _ Rational solutions of the Kadomtsev-Petviashvili equation and integrable systems of $N$ particles on a line, Funct. Anal. Appl. 12 (1978), 59-61.

135. _ Elliptic solutions of the Kadomtsev-Petviashvili equation and integrable systems of particles, Funct. Anal. Appl. 14 (1980), 282-290. MR 82e:58046

136. _ Nonlinear equations and elliptic curves, Revs. Sci. Technology 23 (1983), 51-90. MR 86a: 58044

137. _ Rational solutions of the Zakharov-Shabat equations and completely integrable systems of $N$ particles on a line, J. Sov. Math. 21, 335-345 (1983).

138. _ Elliptic solutions of nonlinear integrable equations and related topics, Acta Appl. Math. 36 (1994), 7-25. MR 95j:58073

139. _ Elliptic solutions to difference non-linear equations and nested Bethe ansatz equations, preprint, solv-int/9804016.

140. I. Krichever, O. Babelon, E. Billey, and M. Talon, Spin generalization of the Calogero-Moser system and the matrix KP equation, Amer. Math. Soc. Transl. (2) 170 (1995), 83-119. MR 96k:58115

141. I. M. Krichever and D. H. Phong, On the integrable geometry of soliton equations and $N=2$ supersymmetric gauge theories, J. Diff. Geom. 45 (1997), 349-389. MR 98b:58078

142. I. Krichever, P. Wiegmann, and A. Zabrodin, Elliptic solutions to difference non-linear equations and related many-body problems, Commun. Math. Phys. 193 (1998), 373-396. CMP 98:13

143. I. Krichever and A. Zabrodin, Spin generalization of the Ruijsenaars-Schneider model, nonabelian 2 D Toda chain and representations of Sklyanin algebra, Russ. Math. Surv. 50:6 (1995), 1101-1150. MR 97f:58068

144. V. B. Kuznetsov, F. W. Nijhoff, and E. K. Sklyanin, Separation of variables for the Ruijsenaars system, Commun. Math. Phys. 189 (1997), 855-877. CMP 98:04

145. P. D. Lax, Integrals of nonlinear equations of evolution and solitary waves, Commun. Math. Phys. 21 (1968), 467-490. MR 38:3620

146. Outline of a theory of the KdV equation, Recent Mathematical Methods in Nonlinear Wave Propagation (ed. by T. Ruggeri), Lecture Notes in Mathematics 1640 (1996), Springer, Berlin, 70-102. CMP 98:07

147. J. E. Lee and M. P. Tsui, The geometry and completeness of the two-phase solutions of the nonlinear Schrödinger equation, Nonlinear Evolution Equations and Dynamical Systems (ed. by S. Carillo and O. Ragnisco), Springer, Berlin, 1990, 94-97. CMP 91:02

148. A. M. Levin and M. A. Olshanetsky, Hierarchies of isomonodromic deformations and Hitchin systems, preprint, hep-th/9709207.

149. A. Liapounoff, Sur une équation transcendante et les équations différentielles linéaires du second ordre à coefficients périodiques, Comptes Rendus 128 (1899), 1085-1088.

150. W. Magnus and S. Winkler, Hill's Equation, Dover, New York, 1979. MR 80k:34001

151. A. Marshakov, On integrable systems and supersymmetric gauge theories, Theoret. Math. Phys. 112 (1997), 791-826. MR 98h:58084

152. V. A. Marchenko, Sturm-Liouville Operators and Applications, Birkhäuser, Basel, 1986. MR 88f:34034

153. A. I. Markushevich, Theory of Functions of a Complex Variable, 2nd. ed., Chelsea, New York, 1985. MR 56:3258

154. V. B. Matveev, Some comments on the rational solutions of the Zakharov-Shabat equations, Lett. Math. Phys. 3 (1979), 503-512. MR 81j:35100

155. V. B. Matveev and A. O. Smirnov, Symmetric reductions of the Riemann $\theta$-function and some of their applications to the Schrödinger and Boussinesq equation, Amer. Math. Soc. Transl. (2) 157 (1993), 227-237. CMP 94:05

156. D. McGarvey, Operators commuting with translations by one. Part I. Representation theorems, J. Math. Anal. Appl. 4 (1962), 366-410. MR 27:594

157. - Operators commuting with translations by one. Part II. Differential operators with periodic coefficients in $L_{p}(-\infty, \infty)$, J. Math. Anal. Appl. 11 (1965), 564-596. MR 35:3483a

158. __ Operators commuting with translations by one. Part III. Perturbation results for periodic differential operators, J. Math. Anal. Appl. 12 (1965), 187-234. MR 35:3483b

159. H. P. McKean and P. van Moerbeke, The spectrum of Hill's equation, Invent. Math. 30 (1975), 217-274. MR 53:936 
160. H. P. McKean and E. Trubowitz, Hill's operator and hyperelliptic function theory in the presence of infinitely many branch points, Commun. Pure Appl. Math. 29 (1976), 143-226. MR 55:761

161. J. Mertsching, Quasi periodic solutions of the nonlinear Schrödinger equation, Fortschr. Phys. 35 (1987), 519-536. MR 89h:35311

162. G. Mittag-Leffler, Sur les équations différentielles linéaires à coefficients doublement périodiques, C. R. Acad. Sci. Paris, 90, 299-300 (1880).

163. R. M. Miura, Korteweg-de Vries equation and generalization, I. A remarkable explicit nonlinear transformation, J. Math. Phys. 9 (1968), 1202-1204. MR 40:6042a

164. R. M. Miura, C. S. Gardner, and M. D. Kruskal, Korteweg-de Vries equation and generalizations. II. Existence of conservation laws and constants of motion, J. Math. Phys. 9 (1968), 1204-1209. MR 40:6042b

165. J. Moser, Three integrable Hamiltonian systems connected with isospectral deformations, Adv. Math. 16 (1975), 197-220. MR 51:12058

166. _ Integrable Hamiltonian systems and spectral theory, Academia Nationale Dei Lincei, Scuola Normale Superiore, Lezione Fermiani, 1983. MR 87j:58042

167. D. Mumford, An algebro-geometric construction of commuting operators and of solutions to the Toda lattice equation, Korteweg de Vries equation and related non-linear equations, Int. Symp. on Algebraic Geometry, Kyoto, 1977, 115-153. MR 83j:14041

168. S. P. Novikov, The periodic problem for the Korteweg-de Vries equation, Funct. Anal. Appl. 8 (1974), 236-246. MR 52:3760

169. S. Novikov, S. V. Manakov, L. P. Pitaevskii, and V. E. Zakharov, Theory of Solitons, Consultants Bureau, New York, 1984. MR 86k:35142

170. M. A. Olshanetsky and A. M. Perelomov, Classical integrable finite-dimensional systems related to Lie Algebras, Phys. Rep. 71 (1981), 313-400. MR 83d:58032

171. A. R. Osborne and G. Boffetta, A summable multiscale expansion for the KdV equation, Nonlinear Evolution Equations: Integrability and Spectral Methods (ed. by A. Degasperis, A. P. Fordy, and M. Lakshmanan), Manchester Univ. Press, Manchester, 1990, 559-569.

172. R. S. Palais, The symmetries of solitons, Bull. Amer. Math. Soc. 34 (1997), 339-403. MR 98f: 58111

173. L. A. Pastur and V. A. Tkachenko, Spectral theory of Schrödinger operators with periodic complex-valued potentials, Funct. Anal. Appl. 22 (1988), 156-158. MR 89d:34056

174. _ An inverse problem for a class of one-dimensional Schrödinger operators with a complex periodic potential, Math. USSR Izv. 37 (1991), 611-629. MR 92c:34099

175. _ Geometry of the spectrum of the one-dimensional Schrödinger equation with a periodic complex-valued potential, Math. Notes 50 (1991), 1045-1050. MR 93h:34147

176. M. V. Pavlov, Nonlinear Schrödinger equation and the Bogolyubov-Whitham method of averaging, Theoret. Math. Phys. 71 (1987), 584-588. MR 89a:35202

177. R. Pego, Origin of the KdV equation, Notices Amer. Math. Soc. 45 (1998), 358.

178. D. Pelinovsky, Rational solutions of the Kadomtsev-Petviashvili hierarchy and the dynamics of their poles.I. New form of a general rational solution, J. Math. Phys. 35 (1994), 5820-5830. MR 95h:58071

179. E. Picard, Sur une généralisation des fonctions périodiques et sur certaines équations différentielles linéaires, C. R. Acad. Sci. Paris 89 (1879), 140-144.

180. _ Sur une classe d'équations différentielles linéaires, C. R. Acad. Sci. Paris 90 (1880), $128-131$.

181. _ Sur les équations différentielles linéaires à coefficients doublement périodiques, J. reine angew. Math. 90 (1881), 281-302.

182. _ Leçons sur Quelques Équations Fonctionnelles, Gauthier Villars, Paris, 1928.

183. E. Previato, The Calogero-Moser-Krichever system and elliptic Boussinesq solitons, in Hamiltonian Systems, Transformation Groups and Spectral Transform Methods (ed. by J. Harnard and J. E. Marsden), CRM, Montréal, 1990, 57-67. MR 92e:58100

184. Monodromy of Boussinesq elliptic operators, Acta Appl. Math. 36 (1994), 49-55. MR 95m:58079

185. _ Seventy years of spectral curves, Integrable Systems and Quantum Groups (ed. by R. Donagi, B. Dubrovin, E. Frenkel, and E. Previato), Lecture Notes in Mathematics 1620, Springer, Berlin, 1996, 419-481. MR 97e:58119 
186. E. Previato and J.-L. Verdier, Boussinesq elliptic solitons: the cyclic case, Proceedings of the Indo-French Conference on Geometry, Dehli, 1993, S. Ramanan and A. Beuaville (eds.), Hindustan Book Agency, Delhi, 1993, 173-185. MR 96f:14038

187. F. S. Rofe-Beketov, The spectrum of non-selfadjoint differential operators with periodic coefficients, Sov. Math. Dokl. 4 (1963), 1563-1566. MR 28:274

188. S. N. M. Ruijsenaars, Complete integrability of relativistic Calogero-Moser systems and elliptic function identities, Commun. Math. Phys. 110 (1987), 191-213. MR 88i:58072

189. J.-J. Sansuc and V. Tkachenko, Spectral properties of non-selfadjoint Hill's operators with smooth potentials, Algebraic and Geometric Methods in Mathematical Physics (ed. by A. Boutel de Monvel and V. Marchenko), Kluwer, Dordrecht, 1996, 371-385. MR 97a:34226

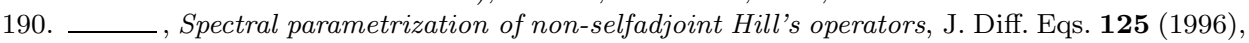
366-384. MR 97a:34222

191. _ Characterization of the periodic and anti-periodic spectra of nonselfadjoint Hill's operators, New Results in Operator Theory and its Applications (ed. by I. Gohberg and Yu. Lubich), Operator Theory: Advances and Applications 98, Birkhäuser, Basel, 1997, 216224. MR 98i:34124

192. J. Schur, Über vertauschbare lineare Differentialausdrücke, Sitzungsber. der Berliner Math. Gesell. 4 (1905), 2-8.

193. G. Segal and G. Wilson, Loop groups and equations of KdV type, Publ. Math. IHES 61 (1985), 5-65. MR 87b:58039

194. T. Shiota, Calogero-Moser hierarchy and KP hierarchy, J. Math. Phys. 35 (1994), 58445849. MR 95i:58095

195. A. O. Smirnov, Elliptic solutions of the Korteweg-de Vries equation, Math. Notes 45 (1989), 476-481. MR 90j:58066

196. _ Real elliptic solutions of the "sine-Gordon" equation, Math. USSR Sbornik /bf 70 (1991), 231-240. MR 92g:14046

197. _ Finite-gap elliptic solutions of the KdV equation, Acta Appl. Math. 36 (1994), 125-166. MR 96c:35173

198. _ Solutions of the KdV equation elliptic in $t$, Theoret. Math. Phys. 100 (1994), 937947. MR 96b:14060

199. _ The Dirac operator with elliptic potential, Sbornik Math. 186 (1995), 1213-1221. MR 96g:35186

200. _ Elliptic solutions of the nonlinear Schrödinger equation and the modified Kortewegde Vries equation, Russ. Acad. Sci. Sb. Math. 82 (1995), 461-470. MR 96f:35157

201. - On a class of elliptic solutions of the Boussinesq equations, Theoret. Math. Phys. 109 (1996), 1515-1522. CMP 98:01

202. $\quad$ The elliptic-in-t solutions of the nonlinear Schrödinger equation, Theoret. Math. Phys. 107 (1996), 568-578. MR 97g:35161

203. - On a class of elliptic potentials of the Dirac operator, Sbornik Math. 188 (1997), 115-135. MR 98e:34153

204. _ Real-valued elliptic solutions of equations related to the sine-Gordon equation, St. Petersburg Math. J. 8 (1997), 513-524. MR 97e:35164

205. - 3-elliptic solutions of the sine-Gordon equation, Math. Notes 62 (1997), 368-376. CMP 98:12

206. V. V. Sokolov, Examples of commutative rings of differential operators, Funct. Anal. Appl. 12 (1978), 65-66. MR 58:17963

207. I. A. Taimanov, Elliptic solutions of nonlinear equations, Theoret. Math. Phys. 84 (1990), 700-706. MR 91k:14020

208. _ On the two-gap elliptic potentials, Acta Appl. Math. 36 (1994), 119-124. MR 95j:33057

209. C.-L. Terng and K. Uhlenbeck, Poisson actions and scattering theory for integrable systems, preprint, dg-ga/9707004.

210. V. A. Tkachenko, Spectral analysis of the one-dimensional Schrödinger operator with periodic complex-valued potential, Sov. Math. Dokl. 5 (1964), 413-415.

211. _ Spectral analysis of a nonselfadjoint Hill operator, Sov. Math. Dokl. 45 (1992), 78-82. MR 93f: 34148

212. , Discriminants and generic spectra of non-selfadjoint Hill's operators, Adv. Sov. Math. 19 (1994), 41-71. MR 95i:34157 
213. _ Spectral properties of periodic Dirac operator with skew-symmetric potential matrix, preprint, 1994.

214. S Spectra of non-selfadjoint Hill's operators and a class of Riemann surfaces, Ann. Math. 143 (1996), 181-231. MR 97f:34067

215. _ Non-selfadjoint periodic Dirac operators, preprint, 1997.

216. _ Non-selfadjoint periodic Dirac operators with finite-band spectrum, preprint, 1998.

217. A. Treibich, Tangential polynomials and elliptic solitons, Duke Math. J. 59 (1989), $611-627$. MR 91k:58059

218. _ Compactified Jacobians of Tangential Covers, Integrable Systems: The Verdier Memorial Conference (ed. by O. Babelon, P. Cartier, Y. Kosmann-Schwarzbach), Birkhäuser, Boston, 1993, 39-60. MR 95k:14043

219. _ Rêvetements tangentiels et condition de Brill-Noether, C. R. Acad. Sci. Paris 316 (1993), 815-817. MR 94b:14023

220. __ New elliptic potentials, Acta Appl. Math. 36 (1994), 27-48. MR 96h:14043

221. _ Matrix elliptic solitons, Duke Math. J. 90 (1997), 523-547. CMP 98:04

222. A. Treibich and J.-L. Verdier, Solitons elliptiques, The Grothendieck Festschrift, Volume III (ed. by P. Cartier, L. Illusie, N. M. Katz, G. Laumon, Y. Manin and K. A. Ribet), Birkhäuser, Basel, 1990, 437-480. MR 92f: 14026

223. _ Revêtements tangentiels et sommes de 4 nombres triangulaires, C. R. Acad. Sci. Paris 311 (1990), 51-54. MR 91k:14022

224. Revêtements exceptionnels et sommes de 4 nombres triangulaires, Duke Math. J. 68 (1992), 217-236. MR 94f:14026

225. _ Variétés de Kritchever des solitons elliptiques de KP, in Proceedings of the IndoFrench Conference on Geometry (Bombay, 1989), Hindustan Book Agency, Delhi, 1993, 187232. MR 95f: 14062

226. Au-delà des potentiels et rêvetements tangentiels hyperelliptiques exceptionnels, C. R. Acad. Sci. Paris 325 (1997), 1101-1106. CMP 98:10

227. A. V. Turbiner, Lame equation, sl(2) algebra and isospectral deformations, J. Phys. A22 (1989), L1-L3. MR 89k:58135

228. K. L. Vaninsky, Trace formula for a system of particles with elliptic potential, preprint, solv-int/9707002.

229. J.-L. Verdier, New elliptic solitons, Algebraic Analysis (ed. by M. Kashiwara and T. Kawai), Academic Press, Boston, 1988, 901-910. MR 90g:58053

230. G. Wallenberg, Über die Vertauschbarkeit homogener linearer Differentialausdrücke, Arch. Math. Phys. 4 (1903), 252-268.

231. R. S. Ward, The Nahm equations, finite-gap potentials and Lamé functions, J. Phys. A20 (1987), 2679-2683. MR 88k:34030

232. R. Weikard, On Hill's equation with a singular complex-valued potential, Proc. London Math. Soc. 76 (1998), 603-633. CMP 98:11

233. - On rational and periodic solutions of stationary KdV equations, preprint 1997.

234. E. T. Whittaker and G. N. Watson, A Course of Modern Analysis, Cambridge University Press, Cambridge, 1986. MR 97k:01072

235. G. Wilson, Commuting flows and conservation laws for Lax equations, Math. Proc. Camb. Phil. Soc. 86 (1979), 131-143. MR 80k:58059

236. _ Algebraic curves and soliton equations, Geometry Today (ed. by E. Arbarello, C. Procesi, and E. Strickland), Birkhäuser, Boston, 1985, 303-329. MR 88i:58077

237. A. Wintner, Stability and spectrum in the wave mechanics of lattices, Phys. Rev. 72 (1947), 81-82. MR 8:615f

238. On the location of continuous spectra, Am. J. Math. 70 (1948), 22-30. MR 9:435k

239. V. A. Yakubovich and V. M. Starzhinskii, Linear Differential Equations with Periodic Coefficients, Vol. 1, Wiley, New York, 1975. MR 51:994

240. N. J. Zabusky and M. D. Kruskal, Interaction of "solitons" in a collisionless plasma and the recurrence of initial states, Phys. Rev. Lett. 15 (1965), 240-243. 
241. V. E. Zakharov and L. D. Faddeev, Korteweg-de Vries equation: A completely integrable Hamiltonian system, Funct. Anal. Appl. 5 (1971), 280-287.

242. V. E. Zakharov and A. S. Shabat, Exact theory of two-dimensional self-focusing and onedimensional self-modulation of waves in nonlinear media, Sov. Phys. JETP 34 (1972), 62-69. MR 53:9966

Department of Mathematics, University of Missouri, Columbia, MO 65211

E-mail address: fritz@math.missouri.edu

$U R L:$ http://www.math.missouri.edu/people/faculty/fgesztesypt.html

Department of Mathematics, University of Alabama at Birmingham, Birmingham, AL 35294-1170

E-mail address: rudi@math.uab.edu

$U R L:$ http://www.math.uab.edu/rudi/ 